\title{
Boundary integral methods in high frequency scattering
}

Book or Report Section

Accepted Version

Postprint version (accepted for publication)

Chandler-Wilde, S. N. and Graham, I. G. (2009) Boundary integral methods in high frequency scattering. In: Engquist, B., Fokas, T., Hairer, E. and Iserles, A. (eds.) Highly Oscillatory Problems. London Mathematical Society Lecture Note Series (366). Cambridge University Press, Cambridge, pp. 154-193. ISBN 9780521134439 Available at http://centaur.reading.ac.uk/1584/

It is advisable to refer to the publisher's version if you intend to cite from the work. See Guidance on citing.

Published version at: http://www.cambridge.org/uk/catalogue/catalogue. asp? isbn=9780521134439

Publisher: Cambridge University Press

All outputs in CentAUR are protected by Intellectual Property Rights law, including copyright law. Copyright and IPR is retained by the creators or other copyright holders. Terms and conditions for use of this material are defined in the End User Agreement. 


\section{www.reading.ac.uk/centaur}

\section{CentAUR}

Central Archive at the University of Reading

Reading's research outputs online 


\title{
Boundary integral methods in high frequency scattering
}

\author{
Simon N. Chandler-Wilde * Ivan G. Graham ${ }^{\dagger}$
}

\begin{abstract}
In this article we review recent progress on the design, analysis and implementation of numerical-asymptotic boundary integral methods for the computation of frequencydomain acoustic scattering in a homogeneous unbounded medium by a bounded obstacle. The main aim of the methods is to allow computation of scattering at arbitrarily high frequency with finite computational resources.
\end{abstract}

\section{Introduction}

There is huge mathematical and engineering interest in acoustic and electromagnetic wave scattering problems, driven by many applications such as modelling radar, sonar, acoustic noise barriers, atmospheric particle scattering, ultrasound and VLSI. For time harmonic problems in infinite domains and media which are predominantly homogeneous, the boundary element method is a very popular solver, used in a number of large commercial codes, see e.g. [26]. In many practical applications the characteristic length scale $L$ of the domain is large compared to the wavelength $\lambda$. Then the small dimensionless wavelength $\lambda / L$ induces oscillatory solutions, and the application of conventional (piecewise polynomial) boundary elements for this multiscale problem yields full matrices of dimension at least $N=(L / \lambda)^{d-1}$ (in $\mathbb{R}^{d}$ ). (Domain finite elements lead to sparse matrices but require even larger $N$.) Since this "loss of robustness" as $L / \lambda \rightarrow \infty$ puts high frequency problems outside the reach of many standard algorithms, much recent research has been devoted to finding more robust methods.

One approach is to seek faster implementations of standard methods. Fast multipole methods have allowed conventional BEM solutions for much larger $N$ (e.g. [28, 29]), but it remains impossible to compute with $L / \lambda$ much beyond a few hundred in $3 \mathrm{D}$. To allow larger $L / \lambda$, a highly promising new direction is the development of "hybrid" algorithms, which incorporate asymptotic information about the oscillation of the solution into the approximation space $[1,2,12,38,50,22,59,6,23,30,13,32,43]$. Initial experiments using geometric-optics type approximations on simple model problems indicate the possibility of delivering almost

*Department of Mathematics, University of Reading, P.O.Box 220, Whiteknights, RG6 6AX, U.K. S.N.Chandler-Wilde@reading. ac.uk

${ }^{\dagger}$ Department of Mathematical Sciences, University of Bath, Bath BA2 7AY, UK. I.G.Graham@bath.ac.uk 
uniform accuracy for $N$ fixed as $L / \lambda \rightarrow \infty$. This review will explain the key ideas behind these methods and the mathematical tools which have been so-far developed for their analysis. We also highlight some important open problems which are the focus of current research in this very active area. Another approach to high frequency problems, involving the solution of appropriate limiting problems, is dealt with elsewhere in this volume [57].

Throughout this review we will focus on the specific physical situation of time harmonic acoustic scattering $\left(\mathrm{e}^{-\mathrm{i} \omega t}\right.$ time dependence for some $\left.\omega>0\right)$; indeed for most of the paper on the case of a sound soft obstacle. This focus is made partly for brevity and simplicity (the algorithms we discuss should generalise to other boundary conditions and to, e.g., elastic and electromagnetic waves), but also because most development of algorithms and most analysis of those algorithms has focused so far on this simplest case. Thus, we suppose an incident plane wave $u^{I}(x)=\exp (\mathrm{i} k x \cdot \hat{a}), x \in \mathbb{R}^{d}$ with direction given by the unit vector $\hat{a}$ and $k$ denoting wavenumber $(k=2 \pi / \lambda=\omega / c$, where $c$ is the wave speed), is scattered by a bounded object $\Omega \subset \mathbb{R}^{d}$ to produce a radiating scattered wave $u^{S}$. The total wave $u=u^{I}+u^{S}$ satisfies the Helmholtz equation:

$$
\Delta u+k^{2} u=0 \quad \text { in } \quad D:=\mathbb{R}^{d} \backslash \Omega \quad(d=2 \text { or } 3) .
$$

Let $\Phi(x, y)$ denote the standard free-space fundamental solution of the Helmholtz equation, given, in the $2 \mathrm{D}$ and $3 \mathrm{D}$ cases, by

$$
\Phi(x, y):= \begin{cases}\frac{\mathrm{i}}{4} H_{0}^{(1)}(k|x-y|), & d=2, \\ \frac{\exp (\mathrm{i} k|x-y|)}{4 \pi|x-y|}, & d=3,\end{cases}
$$

for $x, y \in \mathbb{R}^{d}, x \neq y$, where $H_{\nu}^{(1)}$ denotes the Hankel function of the first kind of order zero. Then in the simplest Dirichlet case $(u=0$ on the boundary $\Gamma)$, starting from Green's representation theorem (see e.g. [23] for details in the general Lipschitz case) we obtain

$$
u(x)=u^{I}(x)-\int_{\Gamma} \Phi(x, y) \frac{\partial u}{\partial n}(y) \mathrm{d} s(y), \quad x \in D,
$$

and the scattering problem can be reformulated as the boundary integral equation (see e.g. $[27])$

$$
\frac{\partial u}{\partial n}(x)+2 \int_{\Gamma}\left(\frac{\partial \Phi(x, y)}{\partial n(x)}-\mathrm{i} \eta \Phi(x, y)\right) \frac{\partial u}{\partial n}(y) \mathrm{d} s(y)=f(x), \quad x \in \Gamma .
$$

Here $\partial / \partial n$ denotes the normal derivative (outward from $\Omega$ ), $\eta>0$ is a coupling parameter (which ensures that (1.4) is well-posed),

$$
f(x):=2 \frac{\partial u^{I}}{\partial n}(x)-2 \mathrm{i} \eta u^{I}(x), \quad x \in \Gamma
$$


and $\partial u / \partial n$ is to be determined. Standard boundary element methods approximate the whole (oscillatory) $\partial u / \partial n$ by (piecewise) polynomials. By contrast the hybrid methods which we shall discuss in the following section employ asymptotic analysis to obtain analytic information about the oscillations in $\partial u / \partial n$. This information is then exploited directly in the numerical method: only slowly-varying components are approximated and this yields a method which is more "robust" as the frequency increases.

Throughout the review we shall make use of the single-layer, double-layer, adjoint doublelayer and hypersingular operators $S, D, D^{\prime}$ and $H$, defined respectively by:

$$
\begin{array}{rlrl}
S \psi & =2 \int_{\Gamma} \Phi(x, y) \psi(y) d s(y), & D \psi & =2 \int_{\Gamma} \frac{\partial \Phi(x, y)}{\partial n(y)} \psi(y) d s(y) \\
D^{\prime} \psi=2 \int_{\Gamma} \frac{\partial \Phi(x, y)}{\partial n(x)} \psi(y) d s(y), & H \psi & =2 \int_{\Gamma} \frac{\partial^{2} \Phi(x, y)}{\partial n(x) \partial n(y)} \psi(y) d s(y) .
\end{array}
$$

The particular equation (1.4) can then be written as

$$
A^{\prime} v:=\left(I+D^{\prime}-\mathrm{i} \eta S\right) v=f, \text { where } v=\partial u / \partial n
$$

This integral equation formulation is well known and is attributed to Burton and Miller in [27]. There one can find a proof that (1.4) is uniquely solvable in $C(\Gamma)$ in the case when $\Gamma$ is sufficiently smooth (a proof of well-posedness in $L^{2}(\Gamma)$, indeed in the Sobolev space $H^{s}(\Gamma$ ) for $-1 \leq s \leq 0$, for the case of general Lipschitz $\Gamma$ is given recently in [23]). It is a direct integral equation formulation, meaning that it arises directly from applying Green's theorems to the solution of the scattering problem, so that the unknown in the integral equation is the unknown part of the Cauchy data of problem (1.1) on the boundary. A closely-related indirect formulation, due to Brakhage \& Werner [11], Leis [53], and Panič [58], obtained by seeking the solution as a linear combination of single- and double-layer potentials with some unknown density $\phi$, can be written in operator form as

$$
A \phi=(I+D-\mathrm{i} \eta S) \phi=-\left.2 u^{I}\right|_{\Gamma} .
$$

Note that equations (1.6) and (1.5) are intimately related; indeed $A^{\prime}$ is the formal adjoint of $A$, as a consequence of which, as operators on $L^{2}(\Gamma), A$ and $A^{\prime}$ have the same spectrum, norm and condition number (see [20]). We shall focus more on (1.5) in this review. As noted by Bruno et al. [12], this equation seems better behaved in the high frequency regime, since its solution is the normal derivative on $\Gamma$ of the solution of the original scattering problem, while it can be shown that the solution $\phi$ of (1.6) is the difference between solutions to interior and exterior boundary value problems. For this reason the solution of (1.5) is less oscillatory and its high frequency behaviour is better understood, especially for convex scatterers.

An important issue for (1.6) and (1.5), which we will address in $\S 3$, is how to choose the coupling parameter $\eta>0$ optimally, e.g. to minimise the condition number of $A^{\prime}$. Discussion of this issue goes back to Kress and Spassow [49] (and see [48, 3, 4, 37, 17, 30, 9, 24, 20]). We will see in $\S 3$ that a correct $k$-dependent choice is essential in the high frequency limit. 
Equation (1.5) is a second-kind integral equation which determines the unknown solution $v:=\frac{\partial u}{\partial n}$, and there is a huge literature on equations of this form. When the boundary $\Gamma$ is sufficiently smooth ( $C^{1}$ is sufficient [33]) the integral operators $D^{\prime}$ and $S$ in (1.5) are compact on standard function spaces, so that $A^{\prime}$ is a compact perturbation of the identity operator. Using classical arguments based on this property, one can show that standard numerical techniques like Galerkin and collocation methods using piecewise polynomial basis functions lead to uniquely determined numerical solutions $v_{N}$ satisfying quasi-optimal error estimates of the form

$$
\left\|v-v_{N}\right\| \leq C \inf _{\phi_{N} \in \mathcal{S}_{N}}\left\|v-\phi_{N}\right\|
$$

where $\mathcal{S}_{N}$ denotes the finite-dimensional approximation space being used (and $N$ is the discretisation parameter, e.g. the dimension of the space $\mathcal{S}_{N}$ ). More precisely, for properlydesigned Galerkin method and collocation methods, these classical arguments (e.g. Atkinson [7]) tell us that there exists a $C>0$ and $N_{0}>0$ such that (1.7) holds for all $N \geq N_{0}$ (see $\S 3.1$ for a little more detail).

Based on (1.7) one can think of the numerical analysis of robust methods for scattering problems as requiring research on three related questions:

Q1 The design of good, $k$-dependent, finite-dimensional approximation spaces $\mathcal{S}_{N}$, so that the best approximation error $\inf _{\phi_{N} \in \mathcal{S}_{N}}\left\|v-\phi_{N}\right\|$ is growing as slowly as possible as $k \rightarrow \infty$. These spaces will normally depend on $k$ and so we denote them $\mathcal{S}_{N, k}$.

Q2 The proof of sharp estimates for the dependence of the "stability constant" $C$ in (1.7) on $k$, hopefully showing that these again indicate boundedness or mild growth as $k \rightarrow \infty$.

Q3 The design of good methods of implementing the numerical methods using the optimal approximation spaces in item 1; ideally show that these are realisable in a computation time which remains bounded as $k \rightarrow \infty$.

For Q1, an "ideal" aim might be that when $v$ is the solution of (1.4), the best approximation error should remain constant for each fixed $N$ as $k \rightarrow \infty$. Recent results on the analysis of this problem are given in $\S 2$.

For Q2, the classical error analysis results for second-kind integral equations tell us that (1.13) holds for all sufficiently large $N\left(N \geq N_{0}\right)$. However, because the wavenumber $k$ appears non-linearly inside the kernel of the operator $A^{\prime}$ in (1.5), they give us no clear quantitative information on either: (i) how, for fixed $N$, the constant $C$ depends on the parameter $k$; or (ii) how, for fixed $C$, the threshold $N_{0}$ depends on $k$. An alternative method of analysis starts from the following variational formulation of (1.5):

$$
\text { Seek } v \in L_{2}(\Gamma) \text { such that } a(v, w)=(f, w)_{L_{2}(\Gamma)} \text {, }
$$

where $a(v, w)=\left(A^{\prime} v, w\right)_{L_{2}(\Gamma)}$. Then the standard abstract theory of variational methods shows, for example, that, provided $a$ satisfies, for all $v, w \in L_{2}(\Gamma)$, the two conditions

$$
\left.\begin{array}{rlr}
|a(v, w)| & \leq B\|v\|_{L_{2}(\Gamma)}\|w\|_{L_{2}(\Gamma)} & \text { (continuity) } \\
|a(v, v)| & \geq \alpha\|v\|_{L_{2}(\Gamma)} & \text { (coercivity) }
\end{array}\right\}
$$


for some positive constants $B$ and $\alpha$, then the equation (1.8) is uniquely solvable. Moreover if the Galerkin (variational) method of approximation is applied to (1.8) in any finite dimensional subspace $\mathcal{S}_{N, k} \subset L_{2}(\Gamma)$, i.e. seek $v_{N} \in \mathcal{S}_{N, k}$ such that

$$
a\left(v_{N}, w_{N}\right)=\left(f, w_{N}\right)_{L_{2}(\Gamma)}, \quad \text { for all } w_{N} \in \mathcal{S}_{N, k},
$$

then we have the error estimate (1.7) with $C=B / \alpha$. Therefore one potential way to answer Q2 is to show that $a$ is coercive and to estimate the dependence of $B$ and $\alpha$ on $k$. Results on this and related problems are discussed in $\S 3$.

Finally, with regard to Q3, in $\S 4$ we discuss recent work on the key implementation issue of computation of the oscillatory integrals which arise in the assembly of stiffness matrices arising from hybrid methods. We also discuss briefly linear algebra issues relating to the fast solution of the dense linear systems arising from hybrid methods.

Before continuing, we would like to explore, a little more carefully, reasonable ways of measuring the accuracy of $v_{N}$. Since $v$ itself depends on $k$, rather than controlling the absolute error in some norm (as in (1.7)), it would seem more sensible to control relative error measures such as

$$
\frac{\left\|v-v_{N}\right\|_{L^{2}(\Gamma)}}{\|v\|_{L^{2}(\Gamma)}} \text { or } \frac{\left\|v-v_{N}\right\|_{L^{2}(\Gamma)}}{\left\|v^{I}\right\|_{L^{2}(\Gamma)}}
$$

where $v^{I}=\partial u^{I} / \partial n$. The attraction of the second of these is that the behaviour of $\left\|v^{I}\right\|_{L^{2}(\Gamma)}$ is clear, in particular it grows proportional to $k$ as $k \rightarrow \infty$ for an arbitrary obstacle. For smooth convex obstacles, for which we know (via the Kirchhoff approximation) that $v \approx 0$ on the shadow side, $v \approx 2 v^{I}$ on the lit side, it is clear that $\|v\|_{L^{2}(\Gamma)}$ grows in proportion to $\left\|v^{I}\right\|_{L^{2}(\Gamma)}$, and so in proportion to $k$. Thus, for the second measure of error, and also for the first in the convex case, controlling the above measures of error, for a fixed obstacle, amounts to controlling

$$
k^{-1}\left\|v-v_{N}\right\|_{L^{2}(\Gamma)} .
$$

A reasonable alternative is to take the view that the computation of $v=\partial u / \partial n$ is an intermediate step, and that the real goal is to compute $u$ accurately in the domain $D$, by substituting the approximation $v_{N}$ to $\partial u / \partial n$ into equation (1.3). Denoting by $u_{N}$ the resulting approximation to $u$, we see that

$$
u(x)-u_{N}(x)=\int_{\Gamma} \Phi(x, y)\left(v_{N}(y)-v(y)\right) \mathrm{d} s(y), \quad x \in D .
$$

In this context we may seek to control

$$
\frac{\left\|u-u_{N}\right\|_{L^{p}(G)}}{\|u\|_{L^{p}(G)}} \text { or } \frac{\left\|u-u_{N}\right\|_{L^{p}(G)}}{\left\|u^{I}\right\|_{L^{p}(G)}},
$$

where $\|\cdot\|_{L^{p}(G)}$, for $1 \leq p \leq \infty$, is the standard $L^{p}$ norm on some region $G \subset D$ (e.g. one might choose $p=2$ or $\infty$, and, in the latter case, choose $G=D$ (as in (2.28) below)). Applying the 
Cauchy-Schwarz inequality to (1.12), we obtain the upper bound:

$$
\left|u(x)-u_{N}(x)\right| \leq c(x)\left\|v-v_{N}\right\|_{L^{2}(\Gamma)}, \quad c(x):=\left\{\int_{\Gamma}|\Phi(x, y)|^{2} \mathrm{~d} s(y)\right\}^{1 / 2} .
$$

Thus small relative error in $u$ can be achieved by controlling $\left\|v-v_{N}\right\|_{L^{2}(\Gamma)}$. However, the value of $\left\|u^{I}\right\|_{L^{p}(G)}$ is independent of $k$, and, in the 3D case, $(c(x))^{2}=(4 \pi)^{-2} \int_{\Gamma}|x-y|^{-2} \mathrm{~d} s(y)$ has a value independent of $k$, while, in $2 \mathrm{D},(c(x))^{2} \sim(\pi /(8 k)) \int_{\Gamma}|x-y|^{-2} \mathrm{~d} s(y)$ as $k \rightarrow \infty$. Thus to achieve small values for the measures of relative error (1.13) by controlling $\left\|v-v_{N}\right\|_{L^{2}(\Gamma)}$ one needs to ensure that

$$
k^{-(3-d) / 2}\left\|v-v_{N}\right\|_{L^{2}(\Gamma)}
$$

is small. Of course, in the high frequency limit, especially in 3D $(d=3)$, this is a significantly stronger requirement than (1.11). We remark that the scaling by $k^{-(3-d) / 2}$ in $(1.15)$ is rather natural in that it makes the expression (1.15) dimensionless.

\section{Hybrid Approximation Spaces}

Instead of approximating $v:=\partial u / \partial n$ in (1.4) directly by piecewise polynomials, the hybrid numerical-asymptotic methods which we are interested in here use approximations with the general form (where we highlight the dependence on $k$ in the notation):

$$
v(x, k) \approx \sum_{m=1}^{M} k \exp \left(\mathrm{i} k \gamma_{m}(x)\right) V_{m}(x, k), \quad x \in \Gamma,
$$

with the phase functions $\gamma_{m}(x)$ chosen a priori and only the unknowns $V_{m}(x, k)$ approximated by piecewise polynomials. The key point is that asymptotic analysis can be used to determine the $\gamma_{m}$ in such a way that the $V_{m}$ are very much less oscillatory than the original $\partial u / \partial n$.

Some of the pioneering work in the development of hybrid boundary element methods for scattering problems was carried out by Abboud et. al. [1, 2], who considered the problem (1.1), subject to the impedance boundary condition : $\frac{\partial u}{\partial n}+i k Z u=0$ on $\Gamma$, and formulated this as the boundary integral equation

$$
-H v+k^{2} Z S(Z v)-\mathrm{i} k D^{\prime}(Z v)-\mathrm{i} k Z D v=g_{k}:=-2 \frac{\partial u_{I}}{\partial n}-2 \mathrm{i} k Z u_{I},
$$

where $v=\left.u\right|_{\Gamma}$, the restriction of $u$ to $\Gamma$. The Galerkin discretisation of this integral equation yields a symmetric stiffness matrix and has no spurious frequencies provided $\Re Z>0$. The authors argued (partly referring to earlier results [40]) that, due to the oscillatory solution of (1.1), in general the conventional boundary element approximation $v_{h}$ of this equation, using step-size $h$ and polynomial degree $p$, would satisfy an error estimate $\left\|v-v_{h}\right\|_{L_{2}(\Gamma)} \leq$ $C(k)(h k)^{p+1}$. Ignoring the unknown factor $C(k)$ this shows that in order to preserve accuracy 
as $k \rightarrow \infty$, we would require $h \sim k^{-1}$ and so, for integral equations on surfaces in $\mathbb{R}^{d}$, the number of degrees of freedom $N$ would have to grow at least with $\mathcal{O}\left(k^{d-1}\right)$. To remedy this, $[1,2]$ suggested taking $M=1$ and $\gamma_{1}(x)=x \cdot \hat{a}$ in $(2.1)$, yielding

$$
v(x, k)=k \exp (\mathrm{i} k x \cdot \hat{a}) V(x, k) \text {, }
$$

and then approximating the unknown "slow variable" $V(\cdot, k)$ using conventional finite element methods. This may be thought of as a numerical implementation of the "Geometric Optics" or "Kirchhoff" approximation which assumes the phase of the scattered wave $u^{S}$ in (1.1) to be the same as the phase of the incoming wave $u^{I}$. (The scaling factor $k$ in (2.2) arises from the differentiation appearing in $v=\partial u / \partial n$.) The function $V(x, k)$ is known to be completely non-oscillatory only in certain regimes (for example when $\Gamma$ is smooth and convex and $x$ is in the illuminated zone and is bounded away from the "shadow boundary" which divides illuminated parts of $\Gamma$ from the parts which are in "shadow"). However for general smooth convex $\Gamma, V(x, k)$ can be expected to be less oscillatory than $v(x, k)$ and it was argued in $[1,2]$ that $\|V(\cdot, k)\|_{H^{n+1}(\Gamma)} \leq C k^{(n+1) / 3}$. (More details of how this estimate can be made rigorous are in $\S 2.1)$. The formal argument sketched above then suggests that using a finite element space of dimension $N=O\left(k^{(d-1) / 3}\right)$ to approximate $V$ in a numerical method based on $(2.2)$ should preserve accuracy as $k \rightarrow \infty$. This, while not being fully "robust" as $k \rightarrow \infty$, is a considerable improvement on the estimate for conventional methods sketched in $\S 1$ although we emphsise that this is not fully rigorous since it ignores the unknown behaviour of $C(k)$.

In more recent work $[12,13]$, Bruno et. al. tackled the breakdown of the geometric optics ansatz by employing a more careful discretisation scheme. Focussing on the formulation (1.4), using also the ansatz (2.2), and multiplying each side of the result by $\exp (-\mathrm{i} k x \cdot \hat{a})$, one obtains

$$
V+\widetilde{D}^{\prime} V-\mathrm{i} \eta \widetilde{S} V=2 \mathrm{i}(k \hat{a} \cdot \hat{n}-\eta) .
$$

The integral operators $\widetilde{D}^{\prime}$ and $\widetilde{S}$ are analogues of $D^{\prime}$ and $S$ with the additional factor $\exp (\mathrm{i} k(y-$ $x) \cdot \hat{a}$ ) in their kernels, leading to kernel functions with easily identified phase. For example,

$$
\begin{aligned}
& \widetilde{S} V(x)=2 \int_{\Gamma} \Phi(x, y) \exp (\mathrm{i} k(y-x) \cdot \hat{a}) V(y) \mathrm{d} y \\
& \quad=\int_{\Gamma} \exp (\mathrm{i} k(|x-y|+(y-x) \cdot \hat{a})) M_{k}(x, y) V(y) \mathrm{d} y,
\end{aligned}
$$

where the factor $M_{k}$ is weakly singular at $y=x$ but is not oscillatory for large $k$.

The approach taken in $[12,13]$ is now to apply a Nyström method to $(2.3)$ based on a suitable quadrature rule for oscillatory integrals of the form (2.4). This involves the approximation of $V$ on a coarse $k$-independent grid. Since (as we shall see more precisely in the following section), the geometric optics approximation breaks down in a boundary layer of width $\mathcal{O}\left(k^{-1 / 3}\right)$ around the shadow boundary, the mesh is graded in $\mathcal{O}\left(k^{-1 / 3}\right)$ neighbourhoods of the shadow boundaries. Based on sampling $V$ at points in this coarse mesh, integration for 
operators such as (2.4) are employed based on partitions of unity and (exponentially convergent) trapezoidal rules. The partition of unity is designed to localise around special points (with respect to the observation point $x$ ) namely (i) the singular point $y=x$; (ii) the stationary points where the gradient of the phase of (2.4) vanishes; (iii) shadow boundary points $n(x) \cdot \hat{a}=0$. As $k \rightarrow \infty$ integration regions become more localised around these points. This is a high-frequency variant of the matrix-free Nyström method of [15]. Since this method is not based on a Galerkin formulation, the analysis of its $k$-robustness is a challenging open problem. We shall return to methods for oscillatory integrals arising in scattering problems in $\S 4$.

A very interesting extension of the method in $[12,13]$ to non-convex scattering is given in [14]. There it is explained how the integral equation (1.4) may be solved by a Neumann series approach, where each term in the Neumann series corresponds to the scattering by a single obstacle of an incident field consisting of the incident wave combined with previously scattered waves. Each of these single-obstacle scattering problems can be solved by a method similar to the methods described above, except that now the ansatz (2.1) becomes somewhat more complicated: the phase $x \cdot \hat{a}$ appearing in (2.2) has to be replaced by a function reflecting the optical distance travelled by rays through all the previous reflections. Preliminary numerical tests were provided in [14] which demonstrated the potential for the method. The theory of this method was substantially advanced in the subsequent work [31, 32]. There the implementation of the Neumann series was shown to correspond to a sum over increasing period of a sequence of periodic orbits. Each orbit corresponds to reflections off a fixed set of scatterers, and this allows the convergence rate of the Neumann series to be estimated, for sufficiently high frequency and permits the formulation of methods for accelerating its convergence. The most recent work in this direction [5] extended the analysis to the three dimensional case, where additional considerations on the relative orientation of the scattering bodies come into play.

In $[31,32,5]$ the emphasis is on the convergence of the Neumann series, and assumes the robust solution of the integral equations arising at each iteration. Thus the proof of the $k$-robustness of the overall algorithm remains a challenging open problem.

One of the substantial challenges which will arise in the rigorous numerical analysis of non-convex scattering problems is that the $k$ - dependence of the constant $C$ in (1.7) is likely to be considerably more complicated than it is in the convex case. We make this statement because $C$ contains in some sense a bound on the inverse operator $A^{-1}$, where $A$ appears in (1.5) (see $\S 3.1$ ). While this inverse is uniformly bounded with respect to $k$ in the convex case (see §3.2), this is not true in the non-convex case. An explicit counter-example is given in [20]. Finally, it is important to point out that, while there has been some progress on aspects of algorithms for the 3D problem (e.g. [14, 36]), the theoretical numerical analysis for this case is limited to date. Moreover the underlying asymptotic theory is much more challenging (e.g. [10] gives a numerical approach to a 3D "canonical problem" and contains extensive references to the asymptotic theory).

In the next two subsections we describe recent work on $2 \mathrm{D}$ problems where more precise rigorous estimates are available, namely scattering by smooth convex obstacles and by convex 
polygons. We note that, in very recent work [51], numerical experiments have been carried out which suggest that the algorithms for these two cases can be successfully combined to compute high frequency scattering by curvilinear convex polygons.

\subsection{The case of smooth $\Gamma$ in $2 \mathrm{D}$}

In this subsection we assume that $\Gamma$ is a $\mathcal{C}^{\infty}$ convex contour. Under plane wave illumination $\Gamma$ is naturally divided by the two tangency points $T_{1}$ and $T_{2}$ into an "illuminated zone" (I) and a "shadow zone" $(\mathbf{S})$, as depicted in Figure 1. Letting $\gamma:[0,2 \pi] \rightarrow \Gamma$ be a $2 \pi$-periodic parametrization of $\Gamma$ we define $t_{i} \in[0,2 \pi)$ to be the preimages of the $T_{i}: \gamma\left(t_{i}\right)=T_{i}$, for $i=1,2$. The "Geometric Optics" ansatz (2.2) may then be written (writing $v(\gamma(s), k)$ as $v(s, k)$ for convenience)

$$
v(s, k)=k \exp (\mathrm{i} k \gamma(s) \cdot \hat{a}) V(s, k) .
$$

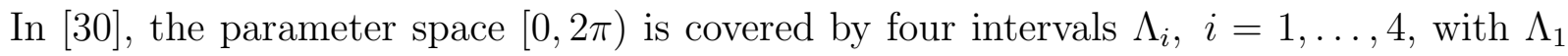
and $\Lambda_{2}$ being suitably small neighbourhoods of the tangency points $t_{1}$ and $t_{2}$, and $\Lambda_{3}$ and $\Lambda_{4}$ contained in the illuminated and shadow zones respectively. Letting $\chi_{i}$ denote a corresponding partition of unity, the $p$-version of the Galerkin method is then applied to (1.8), i.e. we use in (1.10) the approximating space of dimension $N$,

$$
\mathcal{S}_{N, k}:=\operatorname{span}\left\{k \exp (\mathrm{i} k \gamma(s) \cdot \widehat{a}) \chi_{j}(s) P(s): P \in \mathbb{P}_{p_{j}}, j=1,2,3\right\},
$$

where $\mathbb{P}_{p}$ denotes the algebraic polynomials of degree $p$. That is, we use possibly different degree polynomial approximations to $V$ in each of the illuminated and transition zones, and zero approximation to $V$ in the shadow zone.

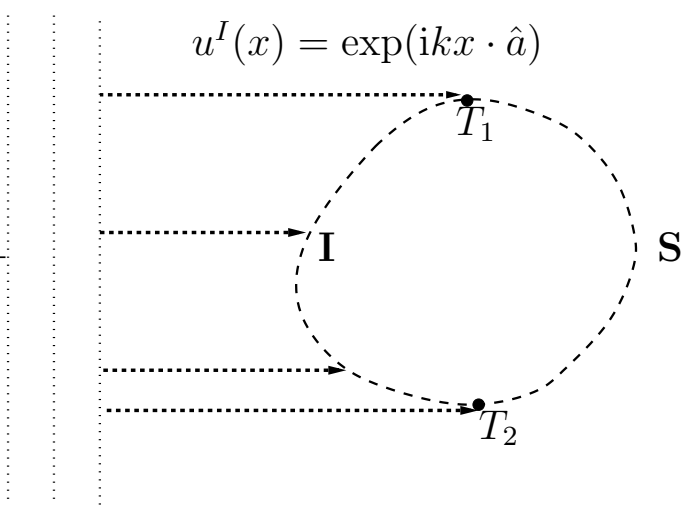

Figure 1: Physical domain: I denotes the illuminated zone, $\mathbf{S}$ the shadow zone and $T_{1}, T_{2}$ the tangency points. 
Considering the numerical analysis of this method we recall the two key questions Q1 and Q2 highlighted in $\S 1$. To answer Q1 we need estimates for the dervatives of $V(s, k)$ with respect to $s$ which are explicit in $k$ in the illuminated and shadow zones. Moreover we need estimates for the exponential decay of $V$ in the deep shadow zone $\Lambda_{1}$. This requires a substantial study of the theory of the "geometric optics" approximation (2.2). In [30] the following result is presented.

Theorem 2.1. For all $L, M \in \mathbb{N} \cup\{0\}$, the function $V(s, k)$ admits a decomposition of the form:

$$
V(s, k)=\left[\sum_{\ell, m=0}^{L, M} k^{-1 / 3-2 \ell / 3-m} b_{\ell, m}(s) \Psi^{(\ell)}\left(k^{1 / 3} Z(s)\right)\right]+R_{L, M}(s, k),
$$

for $s \in[0,2 \pi]$, where the remainder term has its $n$th derivative bounded, for $n \in \mathbb{N} \cup\{0\}$, by

$$
\left|D_{s}^{n} R_{L, M}(s, k)\right| \leq C_{L, M, n}(1+k)^{\mu+n / 3}
$$

where $\mu:=-\min \left\{\frac{2}{3}(L+1),(M+1)\right\}$ and $C_{L . M, n}$ is independent of $k$. The functions $b_{\ell, m}$ and $Z$ are $\mathcal{C}^{\infty} 2 \pi$-periodic functions. $Z$ has simple zeros at $t_{1}$ and $t_{2}$, is positive-valued on $\left[t_{1}, t_{2}\right]$ and negative-valued elsewhere on $[0,2 \pi]$. Moreover $\Psi: \mathbb{C} \rightarrow \mathbb{C}$ is an entire function which may be spcified explicitly by a certain contour integral involving the Airy function (often called "Fock's integral" - see [35, §7, 12], a book originally published in the Russian literature in the late 1940's).

Theorem 2.1 is derived in [30] using the often cited paper [55], combined with the technique of matched asymptotic expansions and also referring to results from the classical literature such as $[18,56,25,19,69,8]$.

The asymptotics of $\Psi(\tau)$ for large $|\tau|$ are of key importance for the behaviour of $V$. Since $Z$ is positive in $\Lambda_{3}$ (inside the illuminated zone), the behaviour of $V(s, k)$ for $s \in \Lambda_{3}$ and for large $k$ is determined by the asymptotics of $\Psi(\tau)$ and its derivatives as $\tau \rightarrow \infty$. Similarly, the behaviour of $V(s, k)$ for $s \in \Lambda_{1}$ (the deep shadow), depends on the asymptotics of $\Psi(\tau)$ for $\tau \rightarrow-\infty$. More complicated behaviour arises in the transition zones $\Lambda_{1}, \Lambda_{2}$. The required properties of $\Psi$ are known. In particular, (see [55, Lemma 9.9]):

$$
\Psi(\tau)=a_{0} \tau+a_{1} \tau^{-2}+a_{2} \tau^{-5}+\ldots+a_{n} \tau^{1-3 n}+\mathcal{O}\left(\tau^{1-3(n+1)}\right), \quad \text { as } \tau \rightarrow \infty,
$$

where $a_{0} \neq 0$ and this expansion remains valid for all derivatives of $\Psi$ by formally differentiating each term on the right hand side, including the error term. Moreover, there exists $\beta>0$ and $c_{0} \neq 0$ such that, for any $n \in \mathbb{N} \cup\{0\}$, as $\tau \rightarrow-\infty$,

$$
D_{\tau}^{n} \Psi(\tau)=c_{0} D_{\tau}^{n}\left\{\exp \left(-\mathrm{i} \tau^{3} / 3-\mathrm{i} \tau \alpha_{1}\right)\right\}(1+\mathcal{O}(\exp (-|\tau| \beta)))
$$

where $\alpha_{1}=\exp (-2 \pi \mathrm{i} / 3) \nu_{1}$ and $\nu_{1}<0$ is the right-most root of the (all real and negative) roots of the Airy function Ai. Hence, when $\tau \rightarrow-\infty$ the function $\Psi$, as well as its derivatives 
decrease exponentially but in a very oscillating way. The asymptotics in (2.10) may be deduced by applying the theory of residues to the contour integral defining $\Psi$ - see [8, p.393], [18, Lemma 8]. More details are in [30]. Combining these asymptotics with Theorem 2.1, the following estimates for the derivatives of $V$ are proved in [30].

Theorem 2.2. For all $n \in \mathbb{N} \cup\{0\}$ there exist constants $C_{n}>0$ independent of $k$ and $s \in[0,2 \pi]$, such that for all $k$ sufficiently large,

$$
\left|D_{s}^{n} V(s, k)\right| \leq C_{n} \begin{cases}1, & n=0,1, \\ k^{-1}\left(k^{-1 / 3}+|\omega(s)|\right)^{-n-2}, & n \geq 2,\end{cases}
$$

where $\omega(s):=\left(s-t_{1}\right)\left(t_{2}-s\right)$. These estimates are uniform in $s \in[0,2 \pi]$.

This statement follows from [30, Theorem 5.4] but is in a somewhat simpler form than given there. The essential point which follows from this is that for $s$ in the illuminated zone and bounded away from $t_{1}, t_{2}$ (and hence $|\omega(s)|$ is bounded away from zero), all derivatives of $V$ are bounded as $k \rightarrow \infty$. This shows the correctness of the Geometric Optics approximation in the interior of the illuminated zone. However, in a region of width $\mathcal{O}\left(k^{-1 / 3}\right)$ around $t_{1}$ or $t_{2}$, $|\omega(s)| \lesssim k^{-1 / 3}$ and $D_{s}^{n} V(s, k)$ may blow up with $\mathcal{O}\left(k^{(n-1) / 3}\right)$. (Notations like $\lesssim$ indicate that there is a hidden constant independent of $k$ ). This corresponds to the estimates employed in the analysis in $[1,2]$ and the motivation for the mesh grading scheme used in [12] which we have described above.

In [30] we considered, for sufficiently large $k>0$ and parameters $\varepsilon, \delta \in(0,1 / 3]$, and $c_{1}>0$, $c_{2}>0$ (to be chosen), the transition zones:

$$
\begin{aligned}
& \Lambda_{1}:=\left[t_{1}-c_{2} k^{-1 / 3+\delta}, t_{1}+c_{1} k^{-1 / 3+\varepsilon}\right], \\
& \Lambda_{2}:=\left[t_{2}-c_{1} k^{-1 / 3+\varepsilon}, t_{2}+c_{2} k^{-1 / 3+\delta}\right],
\end{aligned}
$$

and the illuminated and shadow zones, respectively:

$$
\begin{aligned}
\Lambda_{3} & :=\left[t_{1}+c_{1} k^{-1 / 3+\varepsilon,}, t_{2}-c_{1} k^{-1 / 3+\varepsilon}\right], \\
\Lambda_{4} & :=\left[t_{2}-2 \pi+c_{2} k^{-1 / 3+\delta,}, t_{1}-c_{2} k^{-1 / 3+\delta}\right] .
\end{aligned}
$$

The regions $\Lambda_{j}$ touch only at their endpoints.

In each of the zones $\Lambda_{1}, \Lambda_{2}$ and $\Lambda_{3}$ the error in best approximation by polynomials can be estimated by standard methods. Using Theorem 2.2 and standard error estimates for polynomial approximation it turns out that there are two conflicting choices for $\varepsilon$. The best estimate in the illuminated zone is obtained with $\varepsilon=1 / 3$ (i.e. the boundary of $\Lambda_{1}$ does not approach $t_{1}$ or $t_{2}$ as $k \rightarrow \infty$ ) and the best error in the transition zones is obtained with $\varepsilon=0$ (these zones shrink as fast as possible with $k$ ). To balance the error the best choice turns out to be $\varepsilon=1 / 9$. These estimates have to be combined with the following theorem on exponential decay in the deep shadow which is stated in [30]. 
Theorem 2.3. There exist positive constants $c_{0}, c_{0}^{\prime}$ such that for all $k$ sufficiently large,

$$
\|v\|_{L^{2}\left(\Lambda_{4}\right)} \leq c_{0}^{\prime} \exp \left(-c_{0} k^{\delta}\right)
$$

This result can be formally inferred by using the asymptotics (2.10) in the first term of the right-hand side of (2.7), but this is not a rigorous proof since the remainder term in (2.7) enjoys only algebraically decaying estimates. A brief account of how the proof of Theorem 2.3 follows from the results in the literature is given in [30]. In particular we refer to $[63,34,67,68,52,42,60]$ for the highly non-trivial proofs. An interesting side remark is that the results on exponential decay (in two-dimensional problems) in [67, 68] do not require the contour to be analytic but only sufficiently smooth. There are also extensions to arbitrary dimension, but these require analytic scattering surfaces and (as stated) are only valid in the "deep shadow" (i.e. a bounded distance away from the shadow boundary) - see, e.g. [60, Thm 3] which uses the ideas of [52].

Combining the estimate from Theorem 2.3 with the estimates for polynomial approximation in the illuminated and transition zones, we obtain (in the special case $p_{j}=p$ for each $j=1,2,3$ - see [30] for more general cases), that the Galerkin method solution, defined by (1.10), satisfies the error estimate

$$
\begin{aligned}
\| v & -v_{N}\left\|\leq\left(\frac{B}{\alpha}\right) \inf _{\phi_{N} \in \mathcal{S}_{N, k}}\right\| v(\cdot, k)-\phi_{N} \|_{L^{2}(\Gamma)} \\
& \leq C_{n}\left(\frac{B}{\alpha}\right) k\left\{k^{-4 / 9}\left(\frac{k^{1 / 9}}{p}\right)^{n}+\exp \left(-c_{0} k^{\delta}\right)\right\} .
\end{aligned}
$$

Here (2.13) follows from (1.7) and that, as observed after (1.10), $C \leq B / \alpha$, where $B$ and $\alpha$ are the continuity and coercivity constants from (1.9). Moreover (2.14) follows from the estimates for polynomial approximation and exponential decay described above and holds for all $6 \leq n \leq p+1$ (so in particular, for fixed $k$ and $\mathcal{C}^{\infty}$ data we have superalgebraic convergence, as is normal in the $p$ - version of the boundary element method.)

To make the estimate (2.14) of rigorous use we have to estimate the constant $B$ from above and $\alpha$ from below with respect to $k$. In [30] it is shown that for sufficiently smooth contours $\Gamma$, and in the case $\eta=k$, we have $B \lesssim k^{1 / 2}$. Substantial generalisations of this in [20] are discussed in $\S 3$. Estimates for $\alpha$ from below are much harder. In [30] it was proved by Fourier analysis (on circular or spherical boundaries) that $\alpha \gtrsim 1$ (i.e. $k$-independent coercivity), again for $\eta=k$. However the extension of this result to general $\Gamma$ is a challenging open problem. Neverthess there is hope for success, since in [24] it was proved that $\left\|A^{-1}\right\| \lesssim 1$ for Lipschitz star-shaped $\Gamma$ and any $\eta \in \mathbb{R} \backslash\{0\}$ (where $A$ is the integral operator in (1.5)) and this estimate is a necessary (although not sufficient) condition for $k$-independent coercivity of $a$ defined in (1.8).

On the assumption that $k$-independent coercivity holds for $a$ the result (2.14) shows that the error in the Galerkin approximation is of the order of a low power of $k$ times $\left(k^{1 / 9} / p\right)^{n}$ (for $6 \leq n \leq p+1)$, plus a term which is exponentially small in $k$. Roughly speaking this shows 
that by choosing $p$ to grow slightly faster than $k^{1 / 9}$ we preserve the accuracy of the method as $k$ increases. Numerical results in [30] support this result.

Before leaving this discussion we mention that using the asymptotics (2.10) when $s$ is near to but less than $t_{1}$ (i.e. in the shadow region but near the transition point), then the first term in (2.7) has the asymptotics (as $k \rightarrow \infty)$ :

$$
k^{-1 / 3} b_{0,0} \exp \left(\mathrm{i} k|Z(s)|^{3} / 3\right) \exp \left(\mathrm{i} \Re\left(\alpha_{1}\right) k^{1 / 3}|Z(s)|\right) \exp \left(-\Im\left(\alpha_{1}\right) k^{1 / 3}|Z(s)|\right) .
$$

Since $\alpha_{1}$ is in the first quadrant of the complex plane (see (2.10)), (2.15) contains two oscillatory factors, one oscillating with scale $k$ and one with scale $k^{1 / 3}$, damped by the exponentially decaying third term. These two scales were modelled in the basis functions used in the collocation method of Giladi and Keller, which took into account the existence of "creeping waves" behind the shadow boundary [38].

We now turn our attention to scattering by convex polygonal bodies.

\subsection{The case of polygonal $\Gamma$}

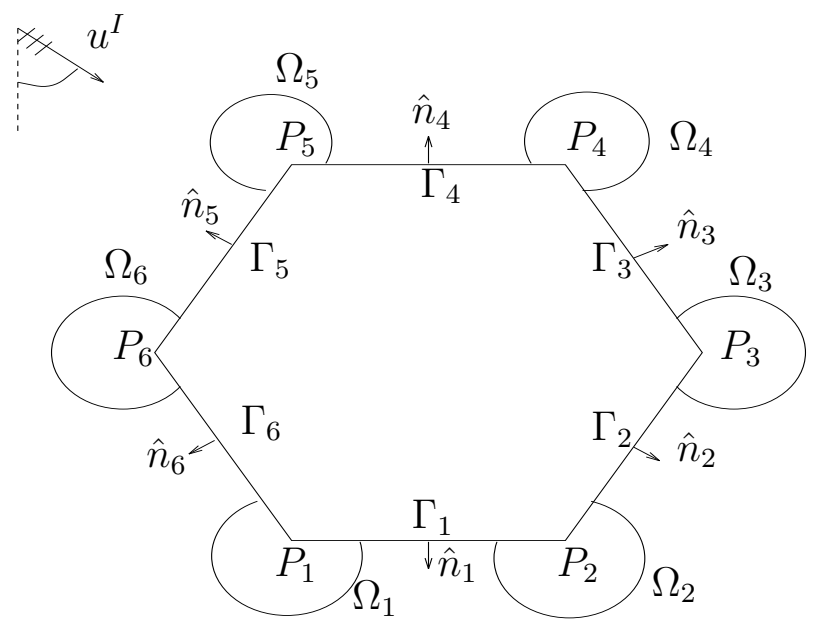

Figure 2: Our notation for the polygon. The $M$ corners are numbered anti-clockwise, and we denote the first corner by both $P_{1}$ and $P_{M+1} . \Omega_{m}$ is the exterior corner angle at $P_{m}$.

In separate work $[6,23]$, scattering by convex polygons, and more recently by curvilinear convex polygons [51], has been considered. For the case of a polygon an appropriate specific form of (2.1) is shown in [23] to be

$$
\begin{aligned}
v(x, k) & \approx k \exp (\mathrm{i} k x \cdot \hat{a}) V(x, k) \\
& +k \sum_{m=1}^{M}\left[\exp \left(\mathrm{i} k x \cdot \hat{a}_{m}\right) V_{m}^{+}(x, k)+\exp \left(-\mathrm{i} k x \cdot \hat{a}_{m}\right) V_{m}^{-}(x, k)\right],
\end{aligned}
$$

for $x \in \Gamma$, where $M$ is the number of sides of the polygon, the unit vector $\hat{a}_{m}$ is parallel to the $m$ th side (directed from corner $P_{m}$ to corner $P_{m+1}$ in Figure 2), and the function $V_{m}^{ \pm}$is 
assumed non-zero only on side $m$. (Physically the terms in the summation are included to represent the parts of the field arising from diffraction at the corners.) In fact, it is shown in [23] that for a polygon it is adequate to take $V(x, k)$ to be constant on each side, precisely to define $V(x, k)$ so that the first term in the above expression is the high frequency Kirchhoff or physical optics approximation, i.e.

$$
\frac{\partial u}{\partial n}(x) \approx\left\{\begin{array}{cc}
2 \frac{\partial u^{I}}{\partial n}(x) & \text { on illuminated sides } \\
0, & \text { on sides in shadow. }
\end{array}\right.
$$

This means to set, for $x \in \Gamma_{m}$,

$$
V(x, k):=\left\{\begin{array}{cc}
2 \mathrm{i} \hat{n}_{m} \cdot \hat{a}, & \text { if } \hat{n}_{m} \cdot \hat{a}<0 \\
0, & \text { otherwise }
\end{array}\right.
$$

where $\hat{n}_{m}$ is the outward unit normal on side $m$. Then, if $V_{m}^{ \pm}$are approximated by piecewise polynomials on carefully chosen graded meshes, uniformly accurate approximations are obtained as $k \rightarrow \infty$, provided the number of degrees of freedom grows like $O\left(\log ^{3 / 2} k\right)$. The results $[6,23]$ are inspired by earlier results $[22,50]$ (indeed the method and analysis for the convex polygon case are outlined in [22]). In these papers, as a prototype of developing and analysing boundary element methods for high frequency scattering, high frequency algorithms are proposed for the problem of $2 \mathrm{D}$ scattering by a flat surface with piecewise continuous impedance boundary condition, and a complete proof of $k$-independent accuracy and complexity is provided, the first such result for any scattering algorithm.

We give now a little more detail of the methods and results of [23]. A key component in the design of the ansatz (2.16) and the design of the graded meshes to approximate the functions $V_{m}^{ \pm}(x, k)$ (both of which are essential to the overall low complexity) is understanding the high frequency behaviour of the solution. We have seen in $\$ 2.1$ that obtaining rigorous results on high frequency asymptotics is, in general, a complex business. But this case of a convex polygon is one in which rigorous high frequency asymptotics, at least a sufficient understanding of these for the purpose of designing an effective numerical scheme, can be achieved by elementary arguments.

The trick (adapted from [22]) is to observe that one can write down an explicit solution to the Dirichlet boundary value problem for the Helmholtz equation in a half-plane, since we (trivially) know by the method of images the Green's function for a half-plane. This observation leads to the following useful representation for the solution to the scattering problem. Let $D_{m} \subset D$ denote the half-plane whose boundary contains $\Gamma_{m}$, the $m$ th side of the polygon (see Figure 3), and let $G_{m}(x, y)$ be the Dirichlet Green's function for the halfplane $D_{m}$, i.e. $G_{m}(x, y)=\Phi(x, y)-\Phi\left(x, y_{m}^{\prime}\right)$, where $y_{m}^{\prime}$ denotes the image of $y$ in the straight line $\partial D_{m}$. Then

$$
u(x)=\epsilon_{m}\left(u^{I}(x)+u^{R}(x)\right)+\int_{\partial D_{m}} \frac{\partial G_{m}(x, y)}{\partial n(y)} u(y) \mathrm{d} s(y), \quad \text { for } \quad x \in D_{m} .
$$




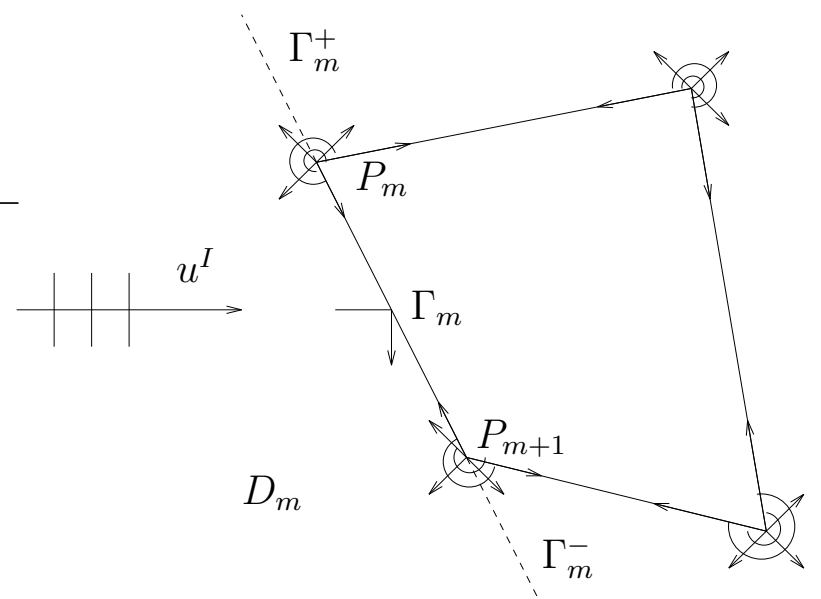

Figure 3: Extension of $\Gamma_{m}$, for derivation of regularity estimates.

Here, $\epsilon_{m}=0$ if side $\Gamma_{m}$ is in shadow, $\epsilon_{m}=1$ if it is illuminated, and $u^{R}$ is the plane wave that would be reflected if $u^{I}$ were incident on the straight line $\partial D_{m}$, on which a Dirichlet boundary condition holds (so $u^{R}(x)=R_{m} \exp \left(\mathrm{i} k x \cdot \hat{a}_{m}^{R}\right)$, where $\hat{a}_{m}^{R}=\hat{a} \cdot \hat{a}_{m} \hat{a}_{m}-\hat{a} \cdot \hat{n}_{m} \hat{n}_{m}$ and the constant $R_{m}$ is chosen so that $u^{I}(x)+u^{R}(x)=0$ on $\Gamma_{m}$; see [23] for details). It is an easy calculation that $\frac{\partial G_{m}(x, y)}{\partial n(y)}=2 \frac{\partial \Phi(x, y)}{\partial n(y)}$, and we recall that $u=0$ on $\Gamma$. Thus, and taking the normal derivative in the above equation, we see that

$$
\frac{\partial u}{\partial n}(x)=2 \epsilon_{m} \frac{\partial u^{I}}{\partial n}(x)+2 \int_{\partial D_{m} \backslash \Gamma_{m}} \frac{\partial^{2} \Phi(x, y)}{\partial n(x) \partial n(y)} u(y) \mathrm{d} s(y), \quad x \in \Gamma_{m} .
$$

It is this equation which justifies the ansatz (2.16), with the explicit formula (2.17) for $V(x, k)$. The first term in the above equation is the Kirchhoff or physical optics approximation and the integral is thus the correction to this approximation on the side $\Gamma_{m}$. To understand the behaviour of this correction we write $\partial D_{m} \backslash \Gamma_{m}$ as the union of the two disjoint half-lines $\Gamma_{m}^{+}$and $\Gamma_{m}^{-}$(see Figure 3). Then we note that, explicitly, for $x \in \Gamma_{m}$ and $y \in \Gamma_{m}^{ \pm}$,

$$
\begin{aligned}
\frac{\partial^{2} \Phi(x, y)}{\partial n(x) \partial n(y)} & =\frac{\mathrm{i} k H_{1}^{(1)}(k|x-y|)}{4|x-y|} \\
& =\frac{\mathrm{i} k^{2}}{4} \exp \left( \pm \mathrm{i} k x \cdot \hat{a}_{m}\right) \exp \left(\mp \mathrm{i} k y \cdot \hat{a}_{m}\right) \mu(k|x-y|),
\end{aligned}
$$

where $\mu(z):=\mathrm{e}^{-\mathrm{i} z} H_{1}^{(1)}(z) / z$, for $z>0$. Thus, we see that, for $x \in \Gamma_{m}$,

$$
\begin{aligned}
v(x, k) & =k \exp (\mathrm{i} k x \cdot \hat{a}) V(x, k) \\
& +k\left[\exp \left(\mathrm{i} k x \cdot \hat{a}_{m}\right) V_{m}^{+}(x, k)+\exp \left(-\mathrm{i} k x \cdot \hat{a}_{m}\right) V_{m}^{-}(x, k)\right],
\end{aligned}
$$

with $V(x, k)$ given by $(2.17)$ and

$$
V_{m}^{ \pm}(x, k):=\frac{\mathrm{i} k}{2} \int_{\Gamma_{m}^{ \pm}} \exp \left(\mp \mathrm{i} k y \cdot \hat{a}_{m}\right) \mu(k|x-y|) u(y) \mathrm{d} s(y) .
$$


The point here is that, while we cannot evaluate the integrals $V_{m}^{ \pm}(x, k)$, because these integrals involve the unknown $u$ on $\Gamma_{m}^{ \pm}$, we can show, in a precise quantitative way, that these functions are not oscillatory on $\Gamma_{m}$. This is the case since $\mu(k|x-y|)$ is very smooth, except when $k|x-y|$ is small, as the function $\mu(z)$, while singular at $z=0$, is increasingly slowly varying as $z \rightarrow \infty$, as quantified in the following lemma from [23].

Lemma 2.4. For every $\epsilon>0$,

$$
\left|\mu^{(m)}(z)\right| \leq\left(1+\epsilon^{-1 / 2}\right)(m+1) ! z^{-3 / 2-m}
$$

for $z \geq \epsilon$ and $m=0,1, \ldots$

Applying this lemma leads to bounds on the tangential derivatives on $\Gamma_{m}$ of $V_{m}^{ \pm}(x, k)$. In this theorem and subsequently it is is convenient to use the abbreviation

$$
u_{M}:=\sup _{x \in D}|u(x)|
$$

to let $L_{m}$ denote the length of $\Gamma_{m}$, and to let $\partial / \partial s$ denote the derivative in the tangential direction on $\Gamma$.

Theorem 2.5. [23, Theorem 3.2] For $x \in \Gamma_{+}$, let $s$ denote the distance of $x$ from the corner $P_{m}$. Then, for $n=0,1, \ldots$, it holds that

$$
\left|\frac{\partial^{n}}{\partial s^{n}} V_{m}^{+}(x, k)\right| \leq 2\left(1+\epsilon^{-1 / 2}\right) u_{M} n ! k^{-1 / 2} s^{-1 / 2-n}, \quad \text { for } \epsilon \leq k s \leq k L_{m} .
$$

The same bound applies to the tangential derivatives of $V_{m}^{-}(x, k)$ but with $s$ denoting distance along $\Gamma_{m}$ from the corner $P_{m+1}$.

This bound captures the behaviour of $V_{m}^{+}(x, k)$ on $\Gamma_{m}$ except near the corner $P_{m}$. But this can be understood by standard elliptic regularity estimates for behaviour of solutions near corners of the domain (e.g. [41]) or more explicitly by writing down a representation for $u$ near the corner $P_{m}$ based on separation of variables in polar coordinates centred on $P_{m}$ [23, theorem 2.3]. The resulting bound is the following, showing that $V_{m}^{+}(x, k)$ has the classic corner singularity behaviour near $P_{m}$ where the exterior corner angle is $\Omega_{m}$.

Theorem 2.6. [23, Corollary 3.4] Suppose that each side of the polygon has length at least $\lambda / 8$, and, for $x \in \Gamma_{+}$, let $s$ denote distance of $x$ from the corner $P_{m}$. Then, for $n=0,1, \ldots$, it holds that

$$
\left|\frac{\partial^{n}}{\partial s^{n}} V_{m}^{+}(x, k)\right| \leq C_{n} u_{M} k^{-\alpha_{m}} s^{-\alpha_{m}-n}, \quad \text { for } 0<k s \leq \pi / 12,
$$

where $\alpha_{m}:=1-\pi / \Omega_{m} \in(0,1 / 2)$, and the value of the constant $C_{n}>0$ depends only on $n$. The same bound applies to the tangential derivatives of $V_{m}^{-}(x, k)$ but with s denoting distance along $\Gamma_{m}$ from the corner $P_{m+1}$, and with $\alpha_{m}$ replaced by $\alpha_{m+1}$. 
The detailed information in the above bounds enables us to construct finite element spaces which are well-adapted to approximating the functions $V_{m}^{ \pm}$. One possibility, used in [23], adapted from [26], is to use a discontinuous piecewise polynomial approximation for each of $V_{m}^{+}$and $V_{m}^{-}$on $\Gamma_{m}$. Thus $V_{m}^{+}$can be approximated using piecewise polynomials of some degree $p$, on a mesh which has a classical grading near the corner $P_{m}$ where $V_{m}^{+}$is singular as quantified in Theorem 2.6, and then has a geometric grading over the rest of $\Gamma_{m}$, with the two meshes joined in a smooth manner. In detail, in the case when each side of the polygon has length $L_{m} \geq \lambda$, defining $q:=(2 p+3) /\left(1-2 \alpha_{m}\right)$, it is shown in [23] that a mesh appropriate for approximating $V_{m}^{+}(x, k)$, given the bounds in the above theorems, consists of the points

$$
s_{i}=\lambda\left(\frac{i}{N}\right)^{q}, i=0, \ldots, N, \text { and } s_{N+j}:=\lambda\left(\frac{L_{m}}{\lambda}\right)^{j / N_{m}^{+}}, j=1, \ldots, N_{m}^{+} .
$$

Here $N$, the number of subintervals in the interval of length $\lambda$ adjacent to the corner $P_{m}$, is the parameter controlling the mesh refinement, and $N_{m}^{+}$is the smallest integer greater than or equal to $-\log \left(L_{m} / \lambda\right) /(q \log (1-1 / N))$. Based on this mesh, an appropriate piecewise polynomial approximation space for $V_{m}^{+}(x, k)$ is

$$
\mathcal{S}_{m}^{+}=\operatorname{span}\left\{\chi_{j}(s) P(s): P \in \mathbb{P}_{p}, j=0,1, \ldots, N+N_{m}^{+}\right\},
$$

where $\chi_{j}(s)$ is the characteristic function of the interval $\left(s_{j-1}, s_{j}\right)$. From the mean value theorem applied to $\log (1-1 / N)$ it follows that the number of subintervals in the geometrically graded part of the mesh satisfies

$$
N_{m}^{+}<q^{-1} N \log \left(L_{m} / \lambda\right)+1
$$

The equations defining a suitable approximation space $\mathcal{S}_{m}^{-}$and mesh for approximating $V_{m}^{-}(x, k)$ are identical to $(2.22)$ and $(2.23)$, except that $q$ is defined replacing $\alpha_{m}$ by $\alpha_{m+1}$ and $s_{i}$ is now the distance of the mesh point from the corner $P_{m+1}$. Since the value of $q$ is different, there is a different number $N_{m}^{-}$of mesh points in the geometrically graded part of the mesh.

Recalling (2.20), we see that an appropriate approximation space for $\partial u / \partial n$ on $\Gamma_{m}$ is

$$
k \exp (\mathrm{i} k x \cdot \hat{a}) V(x, k)+\exp \left(\mathrm{i} k x \cdot \hat{a}_{m}\right) \mathcal{S}_{m}^{+}+\exp \left(-\mathrm{i} k x \cdot \hat{a}_{m}\right) \mathcal{S}_{m}^{-},
$$

with $V(x, k)$ given by $(2.17)$. The full approximation space used in $[6,23]$ is the affine space $\tilde{\mathcal{S}}_{N, k}:=k \exp (\mathrm{i} k x \cdot \hat{a}) V(x, k)+\mathcal{S}_{N, k}$, where $\mathcal{S}_{N, k}$ is the linear space of functions whose restriction to side $\Gamma_{m}$ is in the set $\exp \left(\mathrm{i} k x \cdot \hat{a}_{m}\right) \mathcal{S}_{m}^{+}+\exp \left(-\mathrm{i} k x \cdot \hat{a}_{m}\right) \mathcal{S}_{m}^{-}$, for $m=1, \ldots, M$. The dimension of $\mathcal{S}_{N, k}$, i.e. the number of degrees of freedom, is

$$
\begin{aligned}
D_{N} & =(p+1) \sum_{m=1}^{M}\left(2 N+N_{m}^{+}+N_{m}^{-}\right) \\
& <2 M N\left((p+1)\left(1+N^{-1}\right)+\frac{1}{2} \log (\bar{L} / \lambda)\right)
\end{aligned}
$$

by $(2.24)$, where $\bar{L}:=\left(L_{1} \ldots L_{M}\right)^{1 / M}$.

The main numerical analysis result of [23] is the following best approximation estimate: 
Theorem 2.7. If each side of the polygon has length at least $\lambda$, then, for some positive constant $C_{p}$, depending only on $p$ and on the corner angles $\Omega_{1}, \Omega_{2}, \ldots, \Omega_{M}$, it holds that

$$
k^{-1 / 2} \inf _{\phi_{N} \in \tilde{\mathcal{S}}_{N, k}}\left\|v(\cdot, k)-\phi_{N}\right\|_{L^{2}(\Gamma)} \leq C_{p} u_{M} \frac{(M[1+\log (\bar{L} / \lambda)])^{p+3 / 2}}{D_{N}^{p+1}} .
$$

This theorem shows that, to maintain a given bound on the left hand side of the above inequality, aiming to keep (1.15) fixed as $k$ increases, it is necessary to increase the number of degrees of freedom $D_{N}$ only in proportion to $(\log k)^{3 / 2}$. If one is happy to maintain control instead on $k^{-1} \inf _{\phi \in \mathcal{S}_{N, k}}\|v(\cdot, k)-\phi\|_{L^{2}(\Gamma)}$, aiming to keep (1.13) fixed, then one can even decrease $D_{N}$ as $k$ increases.

In [23] the approximation space $\mathcal{S}_{N, k}$ is used as the basis of a Galerkin method for the integral equation (1.4). Precisely, cf. (1.10), the approximation $v_{N}$ to $v$ is defined by: Seek $v_{N} \in \tilde{\mathcal{S}}_{N, k}$ such that

$$
a\left(v_{N}, w_{N}\right)=\left(f, w_{N}\right)_{L_{2}(\Gamma)}, \quad \text { for all } \quad w_{N} \in \mathcal{S}_{N, k} .
$$

As discussed in $\S 2.1$, in the case that $\Gamma$ is a circle it is shown in [30] that the sesquilinear form $a$ is coercive, with a coercivity constant $\alpha$ independent of $k$. For general domains it is not yet clear whether $a$ is coercive, and in [23] the stability analysis is approached by the classical methods for analysis of second kind equations discussed before equation (1.8) and in §3.1 below. Using these standard methods it is shown in [23] that, for each fixed $k$, there exists a value for the stability constant $C>0$ and an integer $N_{0}$ such that the Galerkin solution is well-defined and (1.7) holds for $N \geq N_{0}$. Combining this with Theorem 2.7 gives the estimate

$$
k^{-1 / 2}\left\|v(\cdot, k)-v_{N}\right\|_{L^{2}(\Gamma)} \leq C C_{p} u_{M} \frac{(M[1+\log (\bar{L} / \lambda)])^{p+3 / 2}}{D_{N}^{p+1}},
$$

for $N \geq N_{0}$, but, as discussed in $\S 1$ and in $\S 3.1$ below, this classical analysis does not give any information about the dependence of $C$ and $N_{0}$ upon $k$. An approximation $u_{N}$ to $u$ can be computed by replacing $v=\partial u / \partial n$ by its the approximation $v_{N}$ in (1.3). This is shown in [23, Theorem 5.4], via the inequality (1.14), to satisfy the error bound

$$
\frac{\sup _{x \in D}\left|u(x)-u_{N}(x)\right|}{\sup _{x \in D}|u(x)|} \leq C C_{p}^{\prime} \frac{(M[1+\log (\bar{L} / \lambda]))^{p+2}}{D_{N}^{p+1}}
$$

for $N \geq N_{0}$, where $C_{p}^{\prime}$ is a positive constant depending only on $p$ and the corner angles $\Omega_{1}$, $\Omega_{2}, \ldots, \Omega_{M}$. Numerical results supporting these error estimates are shown in [23].

A collocation method based on the identical approximation space $\mathcal{S}_{N, k}$ and the identical integral equation formulation is implemented in [6]. No stability analysis (even one based on classical second-kind theory) is made in [6], but the numerical results support the conclusion that there is little difference in accuracy between the Galerkin and the (rather easier to implement) collocation method. 


\section{$3 \quad$ Stability and Conditioning}

\subsection{General considerations}

In $\S 1$ we have split the numerical analysis of high frequency boundary element methods into research on three related questions. We turn in this section to research related to the second of these questions, namely the problem of estimating the value of the stability constant $C$ in (1.7). We note that, while the emphasis of this review is on boundary integral equation methods specifically adapted to high frequency scattering, the results of this section are equally applicable to stability analysis and conditioning for conventional piecewise polynomial boundary element methods at high frequency.

We have noted already that, in the case that the sesquilinear form $a$ is coercive, an upper bound on the stability constant $C$ in the case when $v_{N}$ is defined by the Galerkin method (i.e. by $(1.10))$ is

$$
C \leq \frac{B}{\alpha}
$$

where $B$ and $\alpha$ are the continuity and coercivity constants in (1.9). These constants are closely related to the norms of $A^{\prime}$ and its inverse as operators on $L^{2}(\Gamma)$. Indeed, by Cauchy-Schwarz, for $v, w \in L^{2}(\Gamma)$ (and with $\|\cdot\|$ denoting throughout the norm of a bounded linear operator on $\left.L^{2}(\Gamma)\right)$,

$$
\begin{aligned}
|a(v, w)|=\left|\left(A^{\prime} v, w\right)_{L^{2}(\Gamma)}\right| & \leq\left\|A^{\prime} v\right\|_{L^{2}(\Gamma)}\|w\|_{L^{2}(\Gamma)} \\
& \leq\left\|A^{\prime}\right\|\|v\|_{L^{2}(\Gamma)}\|w\|_{L^{2}(\Gamma)} .
\end{aligned}
$$

Thus $\left\|A^{\prime}\right\|$ is a possible value for the constant $B$ in (1.9). In fact (as follows from setting $w=A^{\prime} v$ in the above inequality), $\left\|A^{\prime}\right\|$ is the smallest possible value for the constant $B$ for which (1.9) holds. Similarly, from the second of the inequalities (1.9),

$$
\left\|A^{\prime} v\right\|_{L^{2}(\Gamma)}\|v\|_{L^{2}(\Gamma)} \geq\left|\left(A^{\prime} v, v\right)_{L^{2}(\Gamma)}\right|=|a(v, v)| \geq \alpha\|v\|_{L^{2}(\Gamma)}^{2},
$$

so that

$$
\left\|A^{\prime-1}\right\| \leq \alpha^{-1}
$$

Thus, the ratio $B / \alpha$ is bounded below by the condition number of the operator $A^{\prime}$ :

$$
\frac{B}{\alpha} \geq \operatorname{cond} A^{\prime}:=\left\|A^{\prime}\right\|\left\|A^{\prime-1}\right\| \text {. }
$$

This gives one motivation for studying the condition number of $A^{\prime}$ and its dependence on $k$, which will be a main topic of this section. Another motivation is the following. The inequality (3.1) is only useful if $a$ is coercive, which we will see below is known to be the case if $\Gamma$ is a circle or sphere, but not, so far, more generally. Whether or not $a$ is coercive, it is 
known that the Galerkin method (1.10) is well-defined if and only if a satisfies the discrete inf-sup condition: that, for some $\gamma_{N}>0$ (the discrete inf-sup constant),

$$
\sup _{0 \neq w \in \mathcal{S}_{N, k}} \frac{|a(v, w)|}{\|w\|_{L^{2}(\Gamma)}} \geq \gamma_{N}\|v\|_{L^{2}(\Gamma)}, \quad \text { for } v \in \mathcal{S}_{N, k} .
$$

If (3.3) holds (which it does, for example, if $a$ is coercive, with $\gamma_{N}=\alpha$ ), then a standard upper bound for the stability constant $C$ is

$$
C \leq 1+\frac{B}{\gamma_{N}}
$$

We do not know of any explicit bounds on the discrete inf-sup constant for high frequency boundary element methods, but we note that $\left\|A^{\prime-1}\right\|=\gamma^{-1}$, where $\gamma$ is the corresponding continuous inf-sup constant, i.e.

$$
\gamma:=\inf _{0 \neq v \in \mathcal{S}_{N, k}} \sup _{0 \neq w \in \mathcal{S}_{N, k}} \frac{|a(v, w)|}{\|v\|_{L^{2}(\Gamma)}\|w\|_{L^{2}(\Gamma)}} .
$$

Thus, if $B$ is the smallest value for which the left-hand inequality in (1.9) holds, then

$$
\text { cond } A^{\prime}=\frac{B}{\gamma} \text {. }
$$

One can hope that studying cond $A^{\prime}$ sheds some light on the behaviour, e.g. as a function of $k$ and the coupling parameter $\eta$, of $B / \gamma_{N}$ and hence of the upper bound (3.4).

As mentioned in $\S 1$, stability can also be studied by classical second-kind integral equation methods [7]: these have the attraction that they also apply to classes of collocation methods, indeed to projection methods in general. Focusing on the Galerkin case, introducing the operator $P_{N}$ of orthogonal projection from $L^{2}(\Gamma)$ to $\mathcal{S}_{N, k}$, and writing $A^{\prime}$ as $A^{\prime}=I+K$, so that $K=D^{\prime}-\mathrm{i} \eta S$, it can be shown (e.g. [7]) that the formulation (1.10) is equivalent to the operator equation

$$
\left(I-P_{N} K\right) v_{N}=P_{N} f
$$

and that the Galerkin method is well-defined if and only if $I-P_{N} K$ is invertible. Moreoever, if the Galerkin method is well-defined, then

$$
v-v_{N}=\left(I-P_{N} K\right)^{-1}\left(v-P_{N} v\right) .
$$

Thus a possible value for the stability constant $C$ in (1.7) is

$$
C=\left\|\left(I-P_{N} K\right)^{-1}\right\|
$$

In the case that $\Gamma$ is $C^{1}$, so that $K$ is compact [33, 27], the classical results tell us that, as long as the spaces $\mathcal{S}_{N}$ have the standard approximation property that $\inf _{\phi_{N} \in \mathcal{S}_{N}}\left\|\phi-\phi_{N}\right\|_{L^{2}(\Gamma)} \rightarrow 0$ as $N \rightarrow \infty$, for every $\phi \in L^{2}(\Gamma)$, then $\left\|P_{N} K-K\right\| \rightarrow 0$ as $N \rightarrow \infty$. Thus

$$
C=\left\|\left(I-P_{N} K\right)^{-1}\right\| \rightarrow\left\|(I-K)^{-1}\right\|=\left\|A^{\prime-1}\right\|
$$


as $N \rightarrow \infty$ (with $k$ fixed). Alternatively, one can write (3.6) as

$$
v-v_{N}=\left(I+L_{N}\right)\left(v-P_{N} v\right), \quad \text { where } L_{N}:=\left(I-P_{N} K\right)^{-1} P_{N} K\left(I-P_{N}\right),
$$

from which equation it is clear that (1.7) holds with

$$
C=1+\left\|L_{N}\right\|
$$

When $K$ is compact it holds that $\left\|L_{N}\right\| \rightarrow 0$ as $N \rightarrow \infty$ (since $\left\|K-K P_{n}\right\| \rightarrow 0$ ). Thus we see that, at least in the case that $\Gamma$ is $C^{1}$, the bound (1.7) holds for every $C>1$ provided $N$ is sufficiently large $\left(N \geq N_{0}\right)$. However, as we have emphasised already in $\S 1$, it is not clear, for a fixed value of $C>1$, how large $N_{0}$ needs to be for (1.7) to hold, and how this value $N_{0}$ depends on $k$. More fundamentally, for Galerkin methods based on hybrid approximation spaces, such as we have discussed in $\S 2$, it is the aim to keep $N$ fixed or almost fixed as $k \rightarrow \infty$, so that it is not clear that we will ever be in the regime where $K P_{N}$ is a good approximation to $K$ in operator norm so that we can prove that $\left\|L_{N}\right\| \approx 0$. On the other hand, it is reasonable to hope that $\left\|\left(I-P_{N} K\right)^{-1}\right\|$ will be well-approximated by $\left\|(I-K)^{-1}\right\|=\left\|A^{\prime-1}\right\|$ before $\left\|P_{N} K-K\right\|$ is small, in which case (1.7) will hold with

$$
C \approx\left\|A^{\prime-1}\right\| \text {. }
$$

We have summarised what the known variational-based and classical second-kind integral equation techniques can tell us about the stability constant $C$. For a recent analysis which is, roughly speaking, intermediate between the two techniques, and its application to the error analysis of conventional boundary integral equation methods at high frequency, see [9].

In the next subsection we discuss what is known about the coercivity constant $\alpha$, the continuity constant $B$ (the smallest choice for which is $\left\|A^{\prime}\right\|$ ), $\left\|A^{\prime-1}\right\|$, and cond $A^{\prime}=\left\|A^{\prime}\right\|\left\|A^{\prime-1}\right\|$, and their dependence on the wavenumber $k$ and the coupling parameter $\eta$ in (1.5).

\subsection{Coercivity and condition numbers}

Studies of the conditioning and spectral properties of integral operators (and their discretisations) in acoustic and electromagnetic scattering date back to Kress and Spassov [49] (and see $[48,3,4,37,66,65,64,17,30,24,20]$ ). Most studies have focussed on the special case when $\Gamma$ is a circle or sphere in which case a very complete theory is possible due to the fact that all the integral operators $S, D, D^{\prime}$ and $H$, defined in $\S 1$, operate diagonally in the basis of trigonometric polynomials $(d=2)$ or spherical harmonics $(d=3)$. The analysis is further simplified by the fact that $D=D^{\prime}$ and so $A=A^{\prime}$ when $\Gamma$ is a circle/sphere.

Suppose $\Gamma$ is the unit circle, with parametrisation $\gamma(s)=(\cos s, \sin s)$. With this parametrisation $L^{2}(\Gamma)$ is isometrically isomorphic to $L^{2}[0,2 \pi]$. We can write any $w \in L^{2}[0,2 \pi]=L^{2}(\Gamma)$ as

$$
w(s)=\frac{1}{2 \pi} \sum_{m \in \mathbb{Z}} \widehat{w}_{m} \exp (\mathrm{i} m s), \quad \text { where } \quad \widehat{w}_{m}:=\int_{0}^{2 \pi} \varphi(s) \exp (-\mathrm{i} m s) \mathrm{d} s,
$$


in which case the $L^{2}$-inner product and norm are given by $(v, w)_{L^{2}(\Gamma)}=\frac{1}{2 \pi} \sum_{m \in \mathbb{Z}} \widehat{v}_{m}{\widehat{\widehat{w}_{m}}}_{\text {and }}$ $\|w\|_{L^{2}(\Gamma)}^{2}=\frac{1}{2 \pi} \sum_{m \in \mathbb{Z}}\left|\widehat{w}_{m}\right|^{2}$. Then (see [48, equation (4.4)] or [30, Lemma 4.1]), we have the Fourier representation:

$$
\begin{aligned}
& A^{\prime} w(s) \quad=\frac{1}{2 \pi} \sum_{m \in \mathbb{Z}} \lambda_{m} \widehat{w}_{m} \exp (\mathrm{i} m s) \\
& \text { with } \quad \lambda_{m}=\pi H_{|m|}^{(1)}(k)\left[\mathrm{i} k J_{|m|}^{\prime}(k)+\eta J_{|m|}(k)\right] .
\end{aligned}
$$

Note that $\lambda_{m}$ is the eigenvalue of $A^{\prime}=A$ corresponding to the eigenfunction $\exp ( \pm \mathrm{i} m s)$. As argued in [48], since the eigenfunctions $\exp (\mathrm{i} m s), m \in \mathbb{Z}$, are a complete orthonormal system in $L^{2}[0,2 \pi]=L^{2}(\Gamma)$, it holds that

$$
\left\|A^{\prime}\right\|=\sup _{m \in \mathbb{N} \cup\{0\}}\left|\lambda_{m}\right|, \quad\left\|A^{\prime-1}\right\|=\left(\inf _{m \in \mathbb{N} \cup\{0\}}\left|\lambda_{m}\right|\right)^{-1},
$$

so that

$$
\text { cond } A^{\prime}=\frac{\sup _{m \in \mathbb{N} \cup\{0\}}\left|\lambda_{m}\right|}{\inf _{m \in \mathbb{N} \cup\{0\}}\left|\lambda_{m}\right|} \text {. }
$$

Further, for $w \in L^{2}(\Gamma)$ we have that

$$
\begin{aligned}
|a(w, w)|=\left|\left(A^{\prime} w, w\right)_{L^{2}(\Gamma)}\right| & \geq \Re\left(A^{\prime} w, w\right)_{L^{2}(\Gamma)} \\
& =\frac{1}{2 \pi} \sum_{m \in \mathbb{Z}} \Re\left(\lambda_{m}\right)\left|\widehat{w}_{m}\right|^{2} \geq \alpha\|w\|_{L^{2}(\Gamma)}^{2}
\end{aligned}
$$

where

$$
\alpha=\inf _{m \in \mathbb{N} \cup\{0\}} \Re\left(\lambda_{m}\right) .
$$

For the case $d=3$, when $\Gamma$ is a sphere of unit radius, a similar analysis applies, based on the fact that the integral operators on the sphere are diagonal operators in the space of spherical harmonics. The corresponding expression for the symbol $\lambda_{m}$ is

$$
\lambda_{m}=\mathrm{i} k h_{m}^{(1)}(k)\left(k j_{m}^{\prime}(k)+\mathrm{i} \eta j_{m}(k)\right)
$$

where $j_{m}$ and $h_{m}^{(1)}$ are the spherical Bessel and Hankel functions respectively. This formula can be found, for example, in [48, 37] - see also [17]. The formulae (3.9), (3.10), and (3.11) hold also in the $3 \mathrm{D}$ case $[48,30]$, with $\lambda_{m}$ given by $(3.12)$.

We see that, for the case of a circle/sphere, studying the coercivity and conditioning of $A^{\prime}$ reduces to the study of the behaviour of explicitly known eigenvalues $\lambda_{m}$, as a function of $m$, the wavenumber $k$, and the coupling parameter $\eta$. The early papers $[49,48]$ carried out a precise theoretical and numerical study of this issue for the case of small $k$, with emphasis on choosing $\eta$ so as to minimise the condition number. The thesis of Giebermann [37] made a similar careful study of the large $k$ case, which is our main focus here, with a mixture of rigorous analysis, indicative asymptotics, and numerical calculation. The substantial gaps in 
the analysis in [37] (in particular, the estimates of the coercivity constant $\alpha$ in [37] were suggestive rather than rigorous: the issue is to obtain sufficiently sharp bounds on the relevant combinations of Bessel functions that are uniform in argument and order) were filled recently in [30], for the explicit choice $\eta=k$ (previously proposed as optimal for conditioning for the unit circle when $k \geq 1$ in e.g. [49, 3, 4]). Further, one of the bounds in [30] was refined recently in [9] (an improved upper-bound on the norm of $D$, the double-layer operator part of $A^{\prime}$ ). Additionally, there are techniques and results that we shall mention below, described in $[24,20]$, that apply to general boundaries, and so to the circle/sphere in particular. The upshot (see [20] for more historical detail) is that the following results are now known for the circle/sphere. In all these bounds $c \geq 1$ denotes some absolute constant, not necessarily the same at each occurrence.

Coercivity for the circle/sphere $([30,20])$. With the choice of coupling parameter $\eta=k$, $\overline{A^{\prime}}=A$ is coercive for all sufficiently large $k$, with $\alpha$ bounded above and below by constants independent of $k$. Indeed, for the circle,

$$
\alpha=1 \text { for all sufficiently large } k \text {. }
$$

Conditioning for the circle/sphere $([30,20])$.

$$
1 \leq\left\|A^{\prime-1}\right\|=\left\|A^{-1}\right\| \leq c\left(1+\frac{1+k}{\eta}\right)
$$

indeed, for a circle and $\eta=k$ it holds that $\left\|A^{\prime-1}\right\|=\left\|A^{-1}\right\|=1$ for all sufficiently large $k$. For a sphere,

$$
1 \leq\left\|A^{\prime}\right\|=\|A\| \leq c\left(1+\eta(1+k)^{-2 / 3}\right) ;
$$

the same bound holds for a circle in the case $\eta=k$. Thus, for a sphere,

$$
\text { cond } A^{\prime}=\text { cond } A \leq c\left(1+k^{1 / 3}\right) \text {, }
$$

if $\eta=1+k^{p}$, for some $p \in[2 / 3,1]$. The same bound holds for a circle, for $k \geq 1$, with the choice $\eta=1+k$.

We note that the above bounds (3.13)-(3.15) suggest that taking $\eta=1+k^{p}$, for some $p \in[2 / 3,1]$ will be approximately optimal in terms of minimising the condition numbers of $A^{\prime}$ and $A$. In fact, for a sphere of radius $R_{0}$, based on low frequency calculations and analysis, the specific choice

$$
\eta=\max \left(\frac{1}{2 R_{0}}, k\right)
$$

was made in [48], and there is further evidence supporting this choice for higher frequencies in $[3,4]$. Recently, Banjai and Sauter [9] have pointed out that, as is clear from (3.15), choosing $\eta=k^{2 / 3}$ gives the same growth rate as $k \rightarrow \infty$ as the choice $\eta=k$, and calculations for the 
case of a circle confirm almost identical values of condition number for $\eta=k / 2$ and $\eta=k^{2 / 3}$ at high wavenumbers.

In recent work by the authors and their collaborators [30, 24, 20, 21] rigorous upper and lower bounds on $\|A\|=\left\|A^{\prime}\right\|$ and $\left\|A^{-1}\right\|=\left\|A^{\prime-1}\right\|$ have been obtained for rather general classes of scatterers, which results show that: (i) the detail of the geometry of $\Gamma$ plays a strong role in determining the dependence of these norms on $k$; (ii) the growth of the condition number with $k$ can be much faster than the mild growth (3.15) for a circle/sphere. We briefly summarise the techniques that have been used and the results that have been obtained.

A first, simple, observation [20, Lemma 4.1] is that both $\left\|A^{\prime}\right\|$ and $\left\|A^{\prime-1}\right\|$ are bounded below by the value 1 , as a consequence of being perturbations of the identity, if some part of $\Gamma$ is at least $C^{1}$ smooth. To obtain upper bounds on $\left\|A^{\prime}\right\|$ rather crude methods are used in [20] which ignore the oscillation in the kernels of the integral operators $D^{\prime}$ and $S$ (whose norms are bounded separately, and then $\left\|A^{\prime}\right\|$ is bounded using the triangle inequality). For example, we bound $\|S\|$ using the estimate

$$
\|S\| \leq 2 \sup _{x \in \Gamma} \int_{\Gamma}|\Phi(x, y)| \mathrm{d} s(y) \leq k^{(d-3) / 2}(2 \pi)^{(1-d) / 2} \int_{\Gamma} \frac{\mathrm{d} s(y)}{|x-y|^{(d-1) / 2}},
$$

the last inequality in fact an equality in the 3D case $(d=3)$. Our resulting bound on the norm of $A^{\prime}$ is the following:

Theorem 3.1. [20, Theorem 3.6] For every Lipschitz $\Gamma$, there exist positive constants $c_{1}$ and $c_{2}$, dependent only on $\Gamma$, such that

$$
\|A\|=\left\|A^{\prime}\right\| \leq 1+c_{1} k^{(d-1) / 2}+c_{2} \eta k^{(d-3) / 2},
$$

for all $k>0$.

In $2 \mathrm{D}(d=2)$, for the case $\Gamma$ simply-connected and smooth, this bound was shown previously, for all sufficiently large $k$, in [30].

We note that these bounds predict, for the usual choice $\eta=k$, a faster growth than (3.14) for a circle/sphere as $k$ increases, namely proportional to $k^{1 / 2}$ in $2 \mathrm{D}, k$ in $3 \mathrm{D}$. Perhaps surprisingly, although the techniques used to obtain the above bounds ignore the increasing oscillation in the kernels of the integral operators as $k$ increases, it is shown in [20] that in $2 \mathrm{D}$ (nothing is known yet about the 3D case) the above bounds are sharp, in the sense that there exist Lipschitz boundaries $\Gamma$ for which $\|S\|$ grows proportional to $k^{-1 / 2}$ and $\left\|D^{\prime}\right\|$ arbitrarily close to $k^{1 / 2}$. In particular:

Lemma 3.2. [20, Theorem 4.2] In the 2D case, if $\Gamma$ contains a straight line section of length $a$, then

$$
\|S\| \geq \sqrt{\frac{a}{\pi k}}+O\left(k^{-1}\right)
$$

as $k \rightarrow \infty$ and

$$
\|A\|=\left\|A^{\prime}\right\| \geq \eta \sqrt{\frac{a}{\pi k}}-1+O\left(\eta k^{-1}\right)
$$


as $k \rightarrow \infty$, uniformly in $\eta>0$.

The quantitative information in the above lemma is pretty sharp. Indeed if $\Gamma$ is a straight line of length $a$ then the formula (3.17) tells us that

$$
\|S\| \leq 2 \sqrt{\frac{a}{\pi k}} .
$$

The technique used to obtain Lemma 3.2 is to construct a $\phi_{k} \in L^{2}(\Gamma)$, dependent on the wavenumber $k$, so as to approximately maximise $\left\|S \phi_{k}\right\|_{L^{2}(\Gamma)} /\left\|\phi_{k}\right\|_{L^{2}(\Gamma)}$. For the proof of Lemma 3.2 the choice $\phi_{k}(x)=\exp (\mathrm{i} k x \cdot \hat{c})$ on the straight line part of $\Gamma$, and zero elsewhere on $\Gamma$, where the unit vector $\hat{c}$ is parallel to the straight line section of $\Gamma$, does the trick.

The same technique can be used to construct lower bounds that explore the subtle interaction between the geometry and the size of $\|S\|$ and $\|D\|=\left\|D^{\prime}\right\|$. For example, one result from [20] is (cf. the bound (3.14) for the case of a circle/sphere):

Lemma 3.3. [20, Corollary 4.5] Suppose (in the $2 D$ case) that $\Gamma$ is locally $C^{2}$ in a neighbourhood of some point $x^{*}$ on the boundary and let $R$ be the radius of curvature at $x^{*}$. If $R<\infty$, then, as $k \rightarrow \infty$,

$$
\|S\| \geq \frac{1}{2}\left(\frac{R}{\pi}\right)^{1 / 3}(2 k)^{-2 / 3}(1+o(1)) .
$$

If also $\eta k^{-2 / 3} \rightarrow \infty$ as $k \rightarrow \infty$, then also

$$
\left\|A^{\prime}\right\|=\|A\| \geq \frac{\eta}{2}\left(\frac{R}{\pi}\right)^{1 / 3}(2 k)^{-2 / 3}(1+o(1)),
$$

as $k \rightarrow \infty$.

Other results in [20] explore what happens if the radius of curvature vanishes (and other higher order smoothness conditions) and under what conditions $\left\|D^{\prime}\right\|=\|D\|$ can be large. The lower bounds in the above lemmas meet the upper bounds in Theorem 3.1 in some cases. For example, if $\Gamma$ is a polygon (as in $\S 2.2$ ) and the usual choice $\eta=k$ is made then, for some constants $c_{1}$ and $c_{2}$,

$$
c_{1} k^{1 / 2} \leq\left\|A^{\prime}\right\|=\|A\| \leq c_{2} k^{1 / 2}
$$

for all sufficiently large $k$. In other cases, for example for an ellipse or some other smooth, strictly convex obstacle, there is a gap between our upper and lower bounds: e.g. for $\eta=k$ our upper bound (Theorem 3.1) gives a growth rate of $k^{1 / 2}$ while our lower bound (Lemma 3.3) has a growth rate of $k^{1 / 3}$. We suspect, from the case of the circle (3.14), and from the evidence of numerical simulations in [21], that it is our lower bounds that are sharp.

One technique that has not been employed yet to obtain upper bounds, which is standard in the harmonic analysis literature [61], and which could be the tool to close the gap, is the observation that, e.g.

$$
\left\|D^{\prime}\right\|=\|D\|=\left\|D D^{*}\right\|^{1 / 2}
$$


Here $D^{*}$ is the Hilbert space adjoint of $D$ (whose kernel is the complex conjugate of the kernel of $D^{\prime}$ ). The point is that $D D^{*}$ is itself an integral operator whose norm can be estimated by the (relatively crude) methods we use to prove Theorem 3.1, and that the kernel of the integral operator $D D^{*}$ is given as an oscillatory integral involving the wavenumber $k$, whose values may be estimated by standard oscillatory integral techniques [61].

To provide upper bounds on $\left\|A^{\prime-1}\right\|=\left\|A^{-1}\right\|$ a completely different technique has been used, namely a priori bounds derived from Rellich-type identities and subtle properties of radiating solutions of the Helmholtz equation [24]. These upper bounds apply for a general class of geometries, namely whenever the scatterer $\Omega$ is simply-connected, piecewise smooth, starlike, and Lipschitz. For the rest of this section we assume, without loss of generality, that the origin lies in $\Omega(0 \in \Omega)$. Then the class of domains studied in [24] are those satisfying the following assumption (Assumption 3 in [24]):

Assumption 3.4. $\Gamma$ is Lipschitz and is $C^{2}$ in a neighbourhood of almost every $x \in \Gamma$. Further

$$
\delta_{-}:=\underset{x \in \Gamma}{\operatorname{essinf}} x \cdot n(x)>0
$$

Note that Assumption 3.4 holds, for example, if $\Omega$ is a convex polyhedron (and $0 \in \Omega$ ), with $\delta_{-}$the distance from the origin to the nearest side of $\Gamma$.

Define

$$
R_{0}:=\sup _{x \in \Gamma}|x|, \quad \delta_{+}:=\operatorname{esssup}_{x \in \Gamma} x \cdot n(x), \quad \delta^{*}:=\operatorname{ess~sup}_{x \in \Gamma}|x-(x \cdot n(x)) n(x)| .
$$

Then a main result in [24] is the following:

Theorem 3.5. Suppose that Assumption 3.4 holds and $\eta>0$. Then

$$
\left\|A^{\prime-1}\right\|=\left\|A^{-1}\right\| \leq B
$$

where $B$ is given by the formula:

$$
\frac{1}{2}+\left[\left(\frac{\delta_{+}}{\delta_{-}}+\frac{4 \delta^{* 2}}{\delta_{-}^{2}}\right)\left[\frac{\delta_{+}}{\delta_{-}}\left(\frac{k^{2}}{\eta^{2}}+1\right)+\frac{d-2}{\delta_{-} \eta}+\frac{\delta^{* 2}}{\delta_{-}^{2}}\right]+\frac{\left(1+2 k R_{0}\right)^{2}}{2 \delta_{-}^{2} \eta^{2}}\right]^{1 / 2} .
$$

To understand this expression for $B$, suppose first that $\Gamma$ is a circle or sphere, i.e. $\Gamma=\{x$ : $\left.|x|=R_{0}\right\}$. Then $\delta_{-}=\delta_{+}=R_{0}$ and $\delta^{*}=0$ so

$$
B=B_{0}:=\frac{1}{2}+\left[1+\frac{k^{2}}{\eta^{2}}+\frac{d-2}{R_{0} \eta}+\frac{\left(1+2 k R_{0}\right)^{2}}{2 R_{0}^{2} \eta^{2}}\right]^{1 / 2} .
$$

In the general case, since $\delta_{-} \leq \delta_{+} \leq R_{0}$ and $0 \leq \delta_{*} \leq R_{0}$, it holds that $B \geq B_{0}$. Note that the expression $B$ blows up if $k / \eta \rightarrow \infty$ or if $\delta_{+} / \delta_{-} \rightarrow \infty$, or if $\delta_{-} \eta \rightarrow 0$, uniformly with respect to the values of other variables. 
An important implication of Theorem 3.5 is that, whenever $\Gamma$ is starlike in the sense of Assumption 3.4, if $\eta$ is chosen so that

$$
\max \left(l_{1} R_{0}^{-1}, l_{2} k\right) \leq \eta \leq \max \left(u_{1} R_{0}^{-1}, u_{2} k\right)
$$

for some positive constants $l_{1}, l_{2}, u_{1}$, and $u_{2}$, then, for some constant $c>0,\left\|A^{\prime-1}\right\|=\left\|A^{-1}\right\| \leq$ $c$, for all $k>0$. For example, choosing

$$
\eta=R_{0}^{-1}+k
$$

which satisfies (3.21) with $l_{1}=l_{2}=1$ and $u_{1}=u_{2}=2$, defining $\theta:=R_{0} / \delta_{-}$, and noting that $\delta_{+} / \delta_{-} \leq \theta, \delta^{*} / \delta_{-} \leq \theta$, we see that Theorem 3.5 implies that

$$
\left\|A^{\prime-1}\right\|=\left\|A^{-1}\right\| \leq B \leq \frac{1}{2}+\theta[2+(1+4 \theta)(d+\theta)]
$$

Based on computational experience, Bruno and Kunyansky [15, 16] recommend the choice $\eta=\max \left(6 T^{-1}, k / \pi\right)$, where $T$ is the diameter of the obstacle, which satisfies (3.21), this formula chosen on the basis of minimising the number of GMRES iterations in an iterative solver. Another choice of $\eta$ satisfying (3.21) is (3.16), recommended as optimal for a sphere for low frequency in [48].

Putting together the bounds of Theorems 3.1 and 3.5, we see that, in the case when $\Gamma$ is piecewise $C^{2}$, Lipschitz and starlike, satisfying Assumption 3.4, it holds, for some constant $c \geq 1$ depending on $\Gamma$, that

$$
1 \leq \mathrm{cond} A^{\prime}=\text { cond } A \leq c\left(1+k^{(d-1) / 2}+\eta k^{(d-3) / 2}\right)\left(1+\frac{1+k}{\eta}\right) .
$$

Thus, for some constant $c^{\prime} \geq 1$,

$$
1 \leq \operatorname{cond} A^{\prime}=\text { cond } A \leq c^{\prime}\left(1+k^{(d-1) / 2}\right),
$$

if $\eta$ is chosen to satisfy (3.21), e.g. given specifically by (3.16) or (3.22).

If $\Gamma$ is not starlike then $\left\|A^{\prime-1}\right\|=\left\|A^{-1}\right\|$ can grow as $k$ increases. In particular, a 2D example is presented in $[20,21]$ in which $\Gamma$ is a trapping-type obstacle, with two straight parallel sides separated by the medium of propagation. It is shown in [20], by combining arguments from [24] with methods of estimating multi-dimensional oscillatory integrals from [47], that, for some constant $c>0$,

$$
\left\|A^{\prime-1}\right\|=\left\|A^{-1}\right\| \geq c k^{9 / 10}(1+\eta / k)^{-1}
$$

and that

$$
\text { cond } A^{\prime}=\text { cond } A \geq c\left(1+k^{14 / 10}\right)
$$

for the usual choice of $\eta$ satisfying (3.21). 


\section{Implementation}

This paper has concentrated on the theory of integral equation formulations for the Helmholtz equation and their numerical solution by Galerkin and collocation methods in the highfrequency case. A hugely important question, which we only have space to deal with briefly, is whether these methods can be realised with computation times which are reasonable as $k \rightarrow \infty$, in particular, do the computation times reflect the theoretical estimates which we have given above? We describe briefly in this section, work on two different issues which are related to this question.

\section{Computation of Oscillatory Stiffness Matrix Entries.}

The Galerkin and collocation methods described above require work on the assembly of stiffness matrices, the entries of which are given as oscillatory integrals. The Nyström approach of Bruno et. al. [12,13] involves a direct approach to the integration problem, without the intermediate step of considering it as part of an expansion method for the integral equation. In any case oscillatory integrals defined on (subsets of) obstacle boundaries with, in addition, weakly singular integrands and complicated phase functions must be computed.

In particular, the hybrid Galerkin discretisation (with (2.1)) of the representative integral operator $v(x) \mapsto \int_{\Gamma} \Phi(x, y) v(y) d s(y)$ taken from (1.4), leads to double integrals of the form

$$
\int_{S_{n^{\prime}}} \int_{S_{n}}\left\{\Phi(x, y) \exp \left(\mathrm{i} k\left[\gamma_{m}(y)-\gamma_{m^{\prime}}(x)\right]\right)\right\} P_{n}(y) P_{n^{\prime}}(x) d s(y) d s(x),
$$

where the $P_{n}$ are (piecewise) polynomial basis functions with supports $S_{n}$. Because the phase of the fundamental solution $\Phi$ is known, the kernel (in the braces) in (4.1) may also be written as $\exp \left(\mathrm{i} k\left[|x-y|+\gamma_{m}(y)-\gamma_{m^{\prime}}(x)\right]\right) K(x, y)$, with $K$ (weakly) singular but non-oscillatory, revealing an oscillatory double integral with a complicated geometry-dependent phase. Collocation methods lead to the simpler (but still oscillatory) single integrals:

$$
\int_{S_{n}}\left\{\Phi(x, y) \exp \left(\mathrm{i} k\left[\gamma_{m}(y)-\gamma_{m^{\prime}}(x)\right]\right)\right\} P_{n}(y) d s(y)
$$

to be evaluated at collocation points $x$.

There has been considerable recent activity on problems of oscillatory integration in general (e.g. [46], [47]), which has provided new insight and analysis for classical methods such as Filon's rule and Levin's method, and, by particularly exploiting asymptotic theory, has also generated new classes of methods. We refer to the separate review [45] in this volume for more detail.

Building on the progress on oscillatory integration in general, Huybrechts and Vandewalle [44] described a general method for computing integrals of the form (4.2) using a numerical variant of the method of steepest descent, in which the integral over $S_{n}$ (parametrised by a real interval) is computed via an integral over a path in the complex plane over which the integrand is not oscillatory. A very nice observation which then follows is that if the collocation point $x$ 
is not in $S_{n}$, if the phase has no stationary points, and if $P_{n}$ has sufficiently many vanishing derivatives then (4.2) vanishes rapidly as $k$ increases. Hence if local basis functions are used in the collocation method, then the collocation matrix can be replaced by a sparse matrix for large $k$. The only non-zero entries of the sparse matrix correspond to points $x$ and supports $S_{n}$ where either $x \in S_{n}$ (a "singular point"), or the phase has a stationary point. Although there is no stability analysis of the method in [44], the numerical results suggest this idea produces a powerful novel algorithm. This idea was further developed in [43, 62] in the context of the partition of unity boundary integral method with plane wave basis functions, applied to general Helmholtz problems (as distinct from the plane wave scattering considered here). In this case the oscillatory integrals can contain very complicated distributions of stationary points.

An application of the method of stationary phase to the computation of collocation matrices arising in boundary integral methods with global basis functions on domains which are diffeomorphic to the sphere is presented in [36]. There the chief difficulty is the problem of locating the stationary points for general geometries.

When choosing quadrature rules for implementing boundary integral methods in the high frequency case, one should bear in mind error estimates for the solution of the integral equation such as (2.14) and Theorem 2.7. It is clear that one requires sharp quadrature error estimates as $N \rightarrow \infty$ with explicit dependence on $k$ in the asymptotic constant. When we apply quadrature to approximate the stiffness matrix for these methods, we only need to ensure that the resulting perturbations satisfy the same kind of error estimates and can then apply classical "Strang Lemma" arguments to obtain error estimates for the whole practical method. Progress on this issue for the 2D Galerkin case on a polygon in the context of $h p$-Galerkin methods has been made by Melenk and Langdon [54]. This approach employs a change of variable of the form $\tau=|x-y|+\gamma_{m}(y)-\gamma_{m^{\prime}}(x)$ for either fixed $x$ or $y$. This ensures that the oscillation in (4.1) is in one variable only, and then applies Filon quadrature. However much work remains to prove rigorous error estimates and extend the results to 3D.

In an intriguing different approach [28] computes integrals such as (4.1) on a subgrid which resolves the oscillations and does this efficiently using a multipole expansion of the kernel factor $\Phi(x, y)$ for $(x, y)$ in each block of a tree-based decomposition of $\Gamma \times \Gamma$. This is a practical alternative to difficult stationary-phase based methods.

Fast methods for dense systems. Matrix compression and fast solvers for non-local operator equations are a major development in numerical analysis in the last 25 years. Typical solvers usually consist of a (preconditioned) Krylov iterative method coupled with a fast matrix-vector multiplication based on kernel approximation (e.g. multipole or panel clustering, or more recently $\mathcal{H}$-matrices). The fast multiplication algorithms work by approximating the (weakly singular) kernel $K(x, y)$ by combinations of separable functions of the form $a_{i}(x) b_{j}(y)$ when $x, y$ are sufficiently separated. Blocks of the dense stiffness matrix are thus replaced by low rank matrices, with the choice of blocking and approximation controlled by a treebased hierarchical algorithm. This allows matrix-vector multiplications with the $N \times N$ dense boundary element stiffness matrix in close to $O(N)$ time. This method has been extended to 
high frequency Helmholtz problems approximated by conventional boundary elements (e.g. $[26,29])$, but the extension to hybrid approximations is an open and fascinating problem. This is important, especially in 3D, since then, even using the hybrid approximation spaces proposed above, $N$ may still be large. The results of [28] show that replacing the Helmholtz kernel with a separable expansion in the far field can still yield low rank approximations even in the hybrid case, but much work remains to be done to yield a solver for which the cost is close to $O(N)$ with a $k$-independent constant.

Acknowledgement: We would like to thank Valery Smyshlyaev for guiding us through the substantial literature in this field from the former Soviet Union.

\section{References}

[1] Abboud, T., Nédélec, J.-C., Zhou, B. (1994). Méthode des équations intégrales pour les hautes fréquencies. C.R. Acad. Sci. Paris. 318 Série I, 165-170.

[2] Abboud, T., Nédélec, J.-C., Zhou, B. (1995). Improvement of the integral equation method for high-frequency problems, in Proceedings of 3rd International Conference on Mathematical Aspects of Wave Propagation Problems (SIAM, Phildelphia).

[3] Amini, S. (1990). On the choice of the coupling parameter in boundary integral formulations of the exterior acoustics problem. Appl. Anal. 35, 75-92.

[4] Amini, S. (1993). Boundary integral solution of the exterior acoustic problem, Comput. Mech. 13, 2-11.

[5] Anand, A., Boubendir, Y., Ecevit, F. and Reitich, F. (2006). Analysis of multiple scattering iterations for high-frequency scattering problems II: The three-dimensional scalar case, report 147, Max-Planck-Institut für Mathematik in den Naturwissenschaften, Leipzig.

[6] Arden, S., Chandler-Wilde, S. N. and Langdon, S. (2007). A collocation method for high frequency scattering by convex polygons. J. Comp. Appl. Math. , 204 334-343.

[7] Atkinson, K. E. (1997). The Numerical Solution of Integral Equations of the Second Kind, (Cambridge University Press, Cambridge).

[8] Babich, V.M., Buldyrev, V.S. (1991). Short-wavelength Diffraction Theory (SpringerVerlag, Berlin).

[9] Banjai, L. and Sauter, S. (2007). A refined Galerkin error and stability analysis for highly indefinite variational problems, SIAM J. Numer. Anal. 45 37-53. 
[10] Bonner, B.D., Graham, I.G. and Smyshlyaev, V.P. (2005). The computation of conical diffraction coefficients in high frequency acoustic wave scattering, SIAM J. Numer. Anal. 43, 1202-1230.

[11] Brakhage, H. and Werner, P. (1965). Über das Dirichletsche Außenraumproblem für die Helmholtzsche Schwingungsgleichung, Arch. Math. 16, 325-329.

[12] Bruno, O.P., Geuzaine, C. A., Monro, J.A. Reitich, F. (2004). Prescribed error tolerances within fixed computational times for scattering problems of arbitrarily high frequency: the convex case. Phil. Trans. R. Soc. Lond. A. 362, 629-645.

[13] Bruno, O.P. and Geuzaine, C. A. (2007). An $\mathcal{O}(1)$ integration scheme for threedimensional surface scattering problems, J. Comp. Appl. Math. , 204, 463-476.

[14] Bruno, O.P., Geuzaine, C.A., Reitich, F. (2005). On the $\mathcal{O}(1)$ solution of multiplescattering problems, IEEE Trans. Magn. 41, 1488-1491.

[15] Bruno, O.P. and Kunyansky, L. (2001). Surface scattering in three dimensions: an accelerated high-order solver, Proc. R. Soc. Lond. A 457, 2921-2934.

[16] Bruno, O.P. (2007). Private communication.

[17] Buffa, A. and Sauter, S. (2006). On the acoustic single layer potential: Stabilisation and Fourier analysis, SIAM J. Sci. Comput. 28, 1974-1999.

[18] Buslaev, V. S. (1964). Short-wave asymptotic behaviour in the problem of diffraction by smooth convex contours (in Russian), Trudy Mat. Inst. Steklov. 73, 14-117. Abbreviated English summary: On the shortwave asymptotic limit in the problem of diffraction by convex bodies, Soviet Physics Doklady 7, 685-687. (1963)

[19] Buslaev, V.S. (1975). The asymptotic behavior of the spectral characteristics of exterior problems for the Schrödinger operator (in Russian). Izv. Akad. Nauk SSSR Ser. Mat. 39, 149-235; English translation (1975). Math. USSR-Izv. 9, 139-223.

[20] Chandler-Wilde, S. N., Graham, I. G., Langdon, S., and Lindner, M. (2007). Condition number estimates for combined potential boundary integral operators in acoustic scattering, Isaac Newton Institute for Mathematical Sciences Preprint NI07067-HOP, Isaac Newton Institute.

[21] Chandler-Wilde, S. N., Graham, I. G., Langdon, S., and Lindner, M. (2007). Condition number estimates for combined potential integral operators in acoustics and their boundary element discretisation, in preparation.

[22] Chandler-Wilde, S. N., Langdon, S. and Ritter, L. (2004). A high-wave number boundary-element method for an acoustic scattering problem, Phil. Trans. R. Soc. Lond. A. 362, 647-671. 
[23] Chandler-Wilde, S. N. and Langdon, S. (2007). A Galerkin boundary element method for high frequency scattering by convex polygons, SIAM J. Numer. Anal. 45, 610-640.

[24] Chandler-Wilde, S. N. and Monk, P. (2008). Wave-number-explicit bounds in timeharmonic scattering, SIAM J. Math. Anal. 39, 1428-1455.

[25] Chazarain, J. (1973). Construction de la paramétrix du problème mixte hyperbolique pour l'equation des ondes, C. R. Acad. Sc. Paris 276, 1213-1215.

[26] Chew, W.C., Song, J.M. , Cui, T.J., Velamparambil, S., Hastriter, and Hu, B. (2004). Review of large scale computing in electromagnetics with fast integral equation solvers, Computer Modeling in Engineering and Sciences, 5, 361-372.

[27] Colton. D. and Kress, R. (1983). Integral Equation Methods in Scattering Theory (Wiley, New York).

[28] Darrigrand, E. (2002). Coupling of fast multipole method and microlocal discretization for the 3-D Helmholtz equation, J. Comput. Phys. 181, 126-154.

[29] Darve, E. and Havé, P. (2004). A fast multipole method for Maxwell equations stable at all frequencies, Phil. Trans. R. Soc. Lond. A, 362, 603-628.

[30] Dominguez, V., Graham, I.G. and Smyshlyaev, V.P. (2007). A hybrid numericalasymptotic boundary integral method for high-frequency acoustic scattering, Numer. Math. 106, 471-510.

[31] Ecevit, F. (2005). Integral equation formulations of electromagnetic and acoustic scattering problems: convergence of multiple scattering iterations and high-frequency asymptotic expansions. PhD Thesis, University of Minnesota.

[32] Ecevit, F., Reitich, F. (2006). Analysis of multiple scattering iterations for high-frequency scattering problems. I: The two dimensional case, report 137, Max-Planck-Institut für Mathematik in den Naturwissenschaften, Leipzig.

[33] Fabes, E. B., Jodeit, M. and Riviere, N.M. (1978). Potential techniques for boundary value problems on $C^{1}$ domains, Acta Math., 141, 165-186.

[34] Filippov, V.B. (1976). Rigorous justification of the shortwave asymptotic theory of diffraction in the shadow zone. J. Sov. Math. 6, 577-626.

[35] Fock, V.A. (1965) Electromagnetic Diffraction and Propagation Problems (Pergamon Press, New York).

[36] Ganesh, M., Langdon, S., Sloan, I.H. (2007). Efficient evaluation of highly oscillatory acoustic scattering surface integrals. J. Comp. Appl. Math. 204, 363-374. 
[37] Giebermann, K. (1997). Schnelle Summationsverfahren zur numerischen Lösung von Integralgleichungen für Streuprobleme im $\mathbb{R}^{3}$. PhD Thesis, University of Karlsruhe.

[38] Giladi, E., Keller, J.B. (2004). An asymptotically derived boundary element method for the Helmholtz equation, in Proceedings of the 20th Annual Review of Progress in Applied Computational Electromagnetics, (Syracuse, New York).

[39] Giladi, E. (2007). Asymptotically derived boundary elements for the Helmholtz equation in high frequencies, J. Comp. Appl. Math., 198, 52 - 74.

[40] Giroire, J. (1982). Integral equation methods for the Helmholtz equation, Integral Equations and Operator Theory 5, 506-517.

[41] Grisvard, P. (1985). Elliptic Problems in Nonsmooth Domains (Pitman, Boston).

[42] Hargé, T., Lebeau, G. (1994). Diffraction par un convexe. Invent. Math. 118, 161-196 .

[43] Honnor, M.E., Trevelyan, J. and Huybrechts, D. (2006). Numerical evaluation of 2D partition of unity boundary integrals for Helmholtz problems, Preprint.

[44] Huybrechts, D. and Vandewalle, S. (2007). A sparse discretisation for integral equation formulations of high-frequency scattering problems, SIAM J. Sci. Comput., 29, 23052328 .

[45] Huybrechts, D. and Olver, S. Highly oscillatory quadrature, this volume.

[46] Iserles, A. and Nørsett, S.P. (2004). On quadrature methods for highly oscillatory integrals and their implementation, BIT 44, 755-772.

[47] Iserles, A. and Nørsett, S.P. (2006). Quadrature methods for multivariate highly oscillatory integrals using derivatives", Math. Comp. 75, 1233-1258.

[48] Kress, R. (1985). Minimizing the condition number of boundary integral operators in acoustic and electromagnetic scattering. Q. Jl. Mech. appl. Math., 38, 323-341.

[49] Kress, R., Spassov, W.T. (1983). On the condition number of boundary integral operators for the exterior Dirichlet problem for the Helmholtz equation, Numer. Math. 42, 77-85.

[50] Langdon, S., Chandler-Wilde, S.N. (2006). A wavenumber independent boundary element method for an acoustic scattering problem, SIAM J. Numer. Anal. 43, 2450-2477.

[51] Langdon, S., Mokgolele. M., Chandler-Wilde, S. N. (2008). High frequency scattering by convex curvilinear polygons, Isaac Newton Institute for Mathematical Sciences Preprint NI08012-HOP.

[52] Lebeau, G. (1984). Régularité Gevrey 3 pour la diffraction, Comm. in Partial Differential Equations 9, 1437-1494. 
[53] Leis, R. (1965). Zur Dirichtletschen Randwertaufgabe des Aussenraums der Schwingungsgleichung, Math. Z. 90, 205-211.

[54] Melenk, J.M. and Langdon, S. (2007). An hp-BEM for high frequency scattering by convex polygons, Proceedings of WAVES2007, University of Reading.

[55] Melrose, R.B., Taylor, M.E. (1985). Near peak scattering and the corrected Kirchhoff approximation for a convex obstacle, Adv. in Math. 55, 242-315.

[56] Morawetz, C.S., Ludwig, D. (1968). An inequality for the reduced wave equation and the justification of geometrical optics, Comm. Pure Appl. Math. 21, 187-203.

[57] Motamed, M. and Runborg, O. (2008). Approximation of high frequency wave propagation problems, this volume.

[58] Panič, O.I. (1965). On the question of the solvability of the exterior boundary-value problems for the wave eqaution and Maxwell's equations, Usp. Mat. Nauk 20A, 221-226.

[59] Perrey-Debain, E., Lagrouche, O., Bettess, P. and Trevelyan, J. (2004). Plane-wave basis finite elements and boundary elements for three-dimensional wave scattering, Phil. Trans. R. Soc. Lond. A, 362, 561-577.

[60] Popov, G. (1987). Some estimates of Green's functions in the shadow, Osaka J. Math. 24, 1-12.

[61] Stein, E. M. (1993). Harmonic Analysis: Real-Variable Methods, Orthogonality, and Oscillatory Integrals (Princeton University Press).

[62] Trevelyan, J. (2007). Numerical steepest descent evaluation of 2D partition of unity boundary integrals for Helmholtz problems, Oberwolfach Reports 5, 354-356.

[63] Ursell, F. (1968). Creeping modes in a shadow, Proc. Camb. Phil. Soc. 68, 171-191.

[64] Warnick, K. F. and Chew, W. C. (2004). Error analysis of the moment method, IEEE Ant. Prop. Mag. 46, 38-53.

[65] Warnick, K. F. and Chew, W. C. (2001). On the spectrum of the electric field integral equation and the convergence of the moment method, Int. J. Numer. Meth. Engng. 51, $31-56$.

[66] Warnick, K. F. and Chew, W. C. (1999). Convergence of moment-method solutions of the electric field integral equation for a 2-D open cavity, Microwave Optical Tech. Letters 23, 212-218.

[67] Zayaev, A.B., Filippov, V.P. (1985). Rigorous justification of the asymptotic solutions of "sliding-wave" type. J. Sov. Math. 30, 2395-2406. 
[68] Zayaev, A.B., Filippov, V.P. (1986). Rigorous justification of the Friedlander-Keller formulas. J. Sov. Math. 32, 134-143.

[69] Zworski, M. (1990). High frequency scatering by a convex obstacle. Duke Math. J. 61, 545-634. 